\title{
Carrier-impurity spin transfer dynamics in paramagnetic II-VI diluted magnetic semiconductors in the presence of a wave-vector-dependent magnetic field
}

\author{
M. Cygorek, ${ }^{1}$ P. I. Tamborenea, ${ }^{2,1}$ and V. M. Axt ${ }^{1}$ \\ ${ }^{1}$ Theoretische Physik III, Universität Bayreuth, 95440 Bayreuth, Germany \\ ${ }^{2}$ Departamento de Física and IFIBA, FCEN, Universidad de Buenos Aires, \\ Ciudad Universitaria, Pabellón I, 1428 Ciudad de Buenos Aires, Argentina
}

\begin{abstract}
Quantum kinetic equations of motion for carrier and impurity spins in paramagnetic II-VI diluted magnetic semiconductors in a k-dependent effective magnetic field are derived, where the carrierimpurity correlations are taken into account. In the Markov limit, rates for the electron-impurity spin transfer can be derived for electron spins parallel and perpendicular to the impurity spins corresponding to measurable decay rates in Kerr experiments in Faraday and Voigt geometry. Our rigorous microscopic quantum kinetic treatment automatically accounts for the fact that, in an individual spin flip-flop scattering process, a spin flip of an electron is necessarily accompanied by a flop of an impurity spin in the opposite direction and the corresponding change of the impurity Zeeman energy influences the final energy of the electron after the scattering event. This shift in the electron energies after a spin flip-flop scattering processes, which usually has been overlooked in the literature, turns out to be especially important in the case of extremely diluted magnetic semiconductors in an external magnetic field. As a specific example for a $\mathbf{k}$-dependent effective magnetic field the effects of a Rashba field on the dynamics of the carrier-impurity correlations in a $\mathrm{Hg}_{1-x-y} \mathrm{Cd}_{y} \mathrm{Mn}_{x} \mathrm{Te}$ quantum well are described. It is found that, although accounting for the Rashba interaction in the dynamics of the correlations leads to a modified $\mathbf{k}$-space dynamics, the time evolution of the total carrier spin is not significantly influenced. Furthermore, a connection between the present theory and the description of collective carrier-impurity precession modes is presented.

PACS numbers: 75.78.Jp, 75.50.Pp, 75.30.Hx, 72.10.Fk
\end{abstract}

\section{INTRODUCTION}

Diluted magnetic semiconductors (DMS) have attracted a great deal of interest ${ }^{1-11}$ as their highly tunable magnetic properties are ideally suited for adding spintronic functionalities to otherwise well-established semiconductor technologies ${ }^{12-14}$. Particularly promising for future technological applications is the fact that some DMS, such as $\mathrm{Ga}_{1-x} \mathrm{Mn}_{x}$ As, exhibit a ferromagnetic phase $^{2,15}$. The convenient optical properties also allow, e.g., for the optical switching of the magnetization ${ }^{16}$ in $\mathrm{Ga}_{1-x} \mathrm{Mn}_{x}$ As. While a comprehensive unified theoretical description of the magnetism in DMS is still missing, it is generally accepted that a carrier-mediated impurityimpurity spin interaction plays a key role ${ }^{1,17}$. Thus, it is crucial to understand the spin physics not only of the magnetic impurities, but also of the carriers as well as the details of the spin transfer between carriers and impurities.

Experimentally, the carrier spins in DMS are often investigated optically using time-resolved magneto-optical Kerr effect (MOKE) measurements ${ }^{3,10,18}$, a pump-probe technique that makes it possible to extract the carrier spin dynamics with a temporal resolution of $\sim 100$ fs. The experimentally obtained carrier spin dephasing and relaxation rates, which also include the effects of the spin transfer between carriers and impurities, can then be used as an input for, e.g., the theoretical description of spin wave excitations in ferromagnetic $\mathrm{DMS}^{8}$.

However, a quantitative theoretical explanation for the values of the carrier spin relaxation rates measured in MOKE experiments, even in the simplest possible case of conduction band electrons in an intrinsic II-VI DMS, has yet to be found. For example, even such basic quantities as the magnetic field dependence of the spin transfer rate between the carrier and impurity systems in paramagnetic DMS is still not satisfactorily explained ${ }^{3}$. This is, on the one hand, due to the large number of factors that simultanously play a role in DMS, like the spin-dependent $s$ - $d$ interaction between magnetic impurities and carriers, spin dephasing due to spin-orbit coupling mechanisms ${ }^{19-21}$, carrier-carrier interaction ${ }^{22}$ and disorder effects ${ }^{23}$. On the other hand, even the typically dominant $s$-d interaction is usually treated only on the level of the mean-field approximation ${ }^{24-26}$, neglecting the effects of carrier-impurity correlations, which can be important ${ }^{8,27,28}$. The spin transfer between carriers and impurities is commonly described by rate equations where the rates are calculated using Fermi's golden rule $^{25,26,29-31}$.

One problem of this approach is that it is a priori not clear under which circumstances the perturbative scheme, which is implicit in the derivation of Fermi's golden rule, is applicable. For example, at the band edge, where the band energies, described by the Hamiltonian $H_{0}$ of an undoped semiconductor, are negligible, the $s$ - $d$ interaction cannot be thought of as a small perturbation to $H_{0}$. A second deficiency of the golden-rule treatment is that it gives, by construction, only the transition rate between energy eigenstates of the system. However, opti- 
cal orientation also allows for an injection of carrier spins perpendicular to an external magnetic field (Voigt geometry) or the impurity magnetization, respectively ${ }^{10}$, which corresponds to the excitation of superpositions of energy eigenstates. Thus, the relaxation rate of the transverse carrier spin component is not provided by Fermi's golden rule.

A more elaborate treatment of the $s$ - $d$ exchange interaction, which is also capable of deriving a rate for the spin transfer of the perpendicular electron spin component, was given by Semenov in a study based on a projection operator method ${ }^{32}$. Another notable approach to the spin dynamics in DMS has been provided by the group of $W u^{22}$, which has developed the kinetic spin Bloch equations (KSBEs) that account not only for rates for the spin transfer due to the $s-d$ exchange interaction, but also for a number of other effects, such as carrier-carrier and carrier-phonon interaction.

In the present article, we describe the electron spin dynamics in the conduction band, where we focus on paramagnetic intrinsic II-VI DMS. We work with a quantum kinetic theory starting from the $s$ - $d$ exchange Hamiltonian $H_{s d}$, where a correlation expansion scheme was used to formulate equations of motion for the carrier and impurity density matrices as well as the carrierimpurity correlation functions ${ }^{33}$. This approach allows a non-perturbative description of far-from-equilibrium situations. The golden-rule rate equations can be deduced from the quantum kinetic theory as a Markovian $\operatorname{limit}^{34,35}$. In the same limit, also the rates for the carrier spin component perpendicular to the impurity magnetization can be derived ${ }^{36}$. Furthermore, the applicability of the Markovian limit and therewith the goldenrule rate equations can be checked by direct comparison of the full quantum kinetic theory with its Markovian $\operatorname{limit}^{36}$. It was found that for an agreement between the quantum kinetic and the Markovian predictions, it is essential to account for a precession-like motion of the carrier-impurity correlations. Therefore, effective equations which capture the essential features of the full quantum kinetic equations that also include the correlation dynamics, were called precession of electron spins and correlations (PESC) equations ${ }^{37}$.

For vanishing external magnetic field and impurity magnetization, all of the above theories contain the same rate equations that can also be found with Fermi's golden rule as a special case. In contrast, in the presence of an external magnetic field which leads to a finite impurity magnetization in the equilibrium of a paramagnetic DMS, the predictions of the different theories deviate from each other. In order to compare these theories, we extend the quantum kinetic theory of Ref. 33 to take into account the Zeeman interaction of carriers and impurities in a magnetic field.

We also allow for a $\mathbf{k}$-dependence of an effective magnetic field, which makes it possible to discuss the effects of Dresselhaus ${ }^{20}$ or Rashba ${ }^{19}$ spin-orbit coupling or a kdependent g-factor on the spin dynamics in DMS. In con- trast to previous treatments ${ }^{21}$ where the PESC equations were extended by adding a $\mathbf{k}$-dependent precession term to the time evolution of the carrier spins, in the present article the $\mathbf{k}$-dependent effective magnetic field is incorporated on a microscopic quantum kinetic level which also leads to a modification of the equations of motion for the carrier-impurity correlations. Another point of view is that, while the approach of Ref. 21 accounts for the k-dependent field between carrier-impurity spin-flip scattering events, in the present theory the effective magnetic field also acts during the spin-flip scattering. Formally this situation is similar to that of, e.g., the intracollisional field effect ${ }^{38}$, where the effects of an external field that acts during a scattering event (phonon-emission in the case of Ref. 38) can indeed change the optical and transport properties qualitatively.

Furthermore, here, we account for the fact that the impurity spin is a $z$-dependent (growth direction of the quantum well) dynamical variable which can change over time. This connects the present theory to the description of collective carrier-impurity precession modes ${ }^{11,39,40}$.

The article is outlined as follows: First, we derive the Markov limit of quantum kinetic equations accounting for the $s$ - $d$ interaction, a possibly k-dependent effective magnetic field and the $z$-dependence of the carrier envelope function. Then, we present results for the magnetic field dependence of the carrier-impurity spin transfer rates and compare it with the results predicted by several other theories. Next, we answer the question to what extent spin-orbit couplings that lead to a k-dependent effective magnetic field influence the spin transfer dynamics, in particular with respect to the dynamics of the carrier-impurity correlations. Finally, we show how the theory of the present paper can be related to the theory employed in the discussion of collective carrier-impurity precession modes ${ }^{39}$.

\section{THEORY}

\section{A. DMS Hamiltonian}

The Hamiltonian for electrons and impurities in DMS can be modelled by

$$
H=H_{0}+H_{Z}^{e}+H_{Z}^{M n}+H_{s d},
$$

where $H_{0}$ describes the conduction band of a semiconductor crystal and can be written as

$$
H_{0}=\sum_{\mathbf{k}} \sum_{\sigma} \hbar \omega_{\mathbf{k}} c_{\mathbf{k} \sigma}^{\dagger} c_{\mathbf{k} \sigma}+\sum_{\mathbf{k}} \sum_{\sigma, \sigma^{\prime}} \hbar \boldsymbol{\Omega}_{\mathbf{k}} \cdot \mathbf{s}_{\sigma \sigma^{\prime}} c_{\mathbf{k} \sigma}^{\dagger} c_{\mathbf{k} \sigma^{\prime}} .
$$

$c_{\mathbf{k} \sigma}^{\dagger}$ and $c_{\mathbf{k} \sigma}$ are the creation and annihilation operators for electrons with wave vector $\mathbf{k}$ in the spin subband $\sigma \in\{\uparrow, \downarrow\} . \omega_{\mathbf{k}}$ describes the diagonal, i.e., the spin independent, part of $H_{0}$ while $\boldsymbol{\Omega}_{\mathbf{k}}$ is the $\mathbf{k}$-dependent effective 
magnetic field, e.g., due to spin-orbit interactions. The electron spin matrix vector $\mathbf{s}_{\sigma \sigma^{\prime}}=\frac{1}{2} \boldsymbol{\sigma}_{\sigma \sigma^{\prime}}$ is proportional to the vector of Pauli matrices $\boldsymbol{\sigma}_{\sigma \sigma^{\prime}}{ }^{41}$.

$H_{Z}^{e}$ and $H_{Z}^{M n}$ are the Zeeman energies for carriers and impurities, respectively:

$$
\begin{aligned}
& H_{Z}^{e}=\sum_{\mathbf{k} \sigma \sigma^{\prime}} g_{e}(\mathbf{k}) \mu_{B} \mathbf{B} \cdot \mathbf{s}_{\sigma \sigma^{\prime}} c_{\mathbf{k} \sigma}^{\dagger} c_{\mathbf{k} \sigma^{\prime}}, \\
& H_{Z}^{M n}=\sum_{I n n^{\prime}} g_{M n} \mu_{B} \mathbf{B} \cdot \mathbf{S}_{n n^{\prime}} \hat{P}_{n n^{\prime}}^{I},
\end{aligned}
$$

where $g_{e}$ and $g_{M n}$ are the electron and impurity g-factors and $\mathbf{B}$ is the externally applied magnetic field. In general, $g_{e}$ may depend on the electron wave vector which, e.g., gives rise to the imhomogeneous-g-factor spin dephasing mechanism ${ }^{42,43}$ which is essential for the description of the magnetic field dependence of the spin decay time in nonmagnetic semiconductors ${ }^{44}$. $\mathbf{S}_{n n^{\prime}}$ are the spin matrices for the impurities with, in the case of Manganese, $\mathrm{S}=\frac{5}{2}$, so that $n, n^{\prime} \in\left\{-\frac{5}{2},-\frac{3}{2}, \ldots, \frac{5}{2}\right\}$. The impurity spin is described by the operator $\hat{P}_{n n^{\prime}}^{I}=|I, n\rangle\left\langle I, n^{\prime}\right|$ where $|I, n\rangle$ is the $n$-th spin state of the $I$-th impurity ion.

The most important part of the Hamiltonian for the spin dynamics in DMS is the $s$ - $d$ exchange interaction which, in real space, has the form:

$$
H_{s d}=J_{s d} \sum_{\substack{I, n, n^{\prime}, i, \sigma, \sigma^{\prime}}}\left(\mathbf{S}_{n n^{\prime}} \hat{P}_{n n^{\prime}}^{I}\right) \cdot \mathbf{s}_{\sigma \sigma^{\prime}} \psi_{\sigma}^{\dagger}\left(\mathbf{r}_{i}\right) \psi_{\sigma^{\prime}}\left(\mathbf{r}_{i}\right) \delta\left(\mathbf{R}_{I}-\mathbf{r}_{i}\right)
$$

where $\mathbf{R}_{I}$ and $\mathbf{r}_{i}$ are the position vectors of the $I$-th impurity and $i$-th electron and $\psi_{\sigma}^{\dagger}\left(\mathbf{r}_{i}\right)$ as well as $\psi_{\sigma}\left(\mathbf{r}_{i}\right)$ are the corresponding real-space field operators for the electrons. Since most experiments on DMS are performed on twodimensional structures, we choose a single-particle basis comprised of product states of a $z$-dependent envelope, where $z$ is defined to point along the growth direction, and an in-plane part described by plane waves. When restricting to the lowest confined state of the envelope function $\psi(z)$, we can formulate the effective $s$ - $d$ Hamiltonian for the in-plane part as:

$H_{s d}=\frac{J_{s d}}{V} d \sum_{I}\left|\psi\left(Z_{I}\right)\right|^{2} \mathbf{S}_{n n^{\prime}} \cdot \mathbf{s}_{\sigma \sigma^{\prime}} c_{\mathbf{k} \sigma}^{\dagger} c_{\mathbf{k}^{\prime} \sigma^{\prime}} \hat{P}_{n n^{\prime}}^{I} e^{i\left(\mathbf{k}^{\prime}-\mathbf{k}\right) \mathbf{R}_{I}^{\|}}$

where $V$ is the volume of the sample, $d$ is the quantum well width, $Z_{I}$ is the z-component of the $I$-th impurity position vector and $\mathbf{R}_{I}^{\|}$is the in-plane part of the position vector of the $I$-th impurity. Assuming infinitely high barriers, the envelope is given by

$$
\psi(z)=\sqrt{\frac{2}{d}} \cos \left(\frac{\pi}{d} z\right),
$$

for $z \in\left[-\frac{d}{2} ; \frac{d}{2}\right]$ and zero otherwise. Thus, due to the factor $\left|\psi\left(Z_{I}\right)\right|^{2}$, magnetic impurities at the border of the quantum well couple much more weakly to the electrons than impurities at the center of the well.

\section{B. Quantum Kinetic Equations of Motion}

In Ref. 33, a set of quantum kinetic equations of motion based on a correlation expansion scheme has been developed for the carrier and impurity density matrix as well as the carrier-impurity correlations in the case of zero external and effective-spin-orbit magnetic fields. In the present article, we additionally consider an in general wave-vector dependent effective magnetic field for the carriers and the Zeeman energy term for the magnetic impurities to the Hamiltonian. Since all of the terms that are added are effective single-particle contributions, they do not lead to a build-up of a new hierarchy of correlations, but only connect the density matrices and the correlations with themselves. Therefore, the trucation scheme and the factorization of higher correlations layed out in Ref. 33 can still be applied when the aforementioned additional Hamiltonians are accounted for. If an on average homogeneous distribution of magnetic impurities in the quantum-well plane is assumed, equations of motion can be formulated for the dynamical variables ${ }^{36}$

$$
\begin{aligned}
C_{\sigma_{1} \mathbf{k}_{1}}^{\sigma_{2}} & =\left\langle c_{\mathbf{k}_{1} \sigma_{1}}^{\dagger} c_{\mathbf{k}_{1} \sigma_{2}}\right\rangle, \\
M_{n_{1}}^{n_{2}}(z)= & \frac{d}{N_{M n}} \sum_{I} \delta\left(z-Z_{I}\right)\left\langle\hat{P}_{n_{1} n_{2}}^{I}\right\rangle, \\
Q_{\sigma_{1} n_{1} \mathbf{k}_{1}}^{\sigma_{2} n_{2} \mathbf{k}_{2}}(z)= & \frac{V}{N_{M n}} d \sum_{I} \delta\left(z-Z_{I}\right) \times \\
& \left\langle c_{\mathbf{k}_{1} \sigma_{1}}^{\dagger} c_{\mathbf{k}_{2} \sigma_{2}} \hat{P}_{n_{1} n_{2}}^{I} e^{\left.i\left(\mathbf{k}_{2}-\mathbf{k}_{1}\right) \mathbf{R}_{I}^{\|}\right\rangle}\right.
\end{aligned}
$$

where $C_{\sigma_{1} \mathbf{k}_{1}}^{\sigma_{2}}$ and $M_{n_{1}}^{n_{2}}(z)$ are the electron and impurity density matrices and $Q_{l_{1} n_{1} \mathbf{k}_{1}}^{l_{2} n_{2} \mathbf{k}_{2}}(z)$ (for $\mathbf{k}_{1} \neq \mathbf{k}_{2}$ ) represent the carrier-impurity correlations, where the mean-field part has been subtracted. $N_{M n}$ is the number of impurity ions in the DMS.

Instead of the density matrices, also the average carrier $\mathbf{s}_{\mathbf{k}_{1}}$ and impurity spins $\langle\mathbf{S}(z)\rangle$ as well as the electron occupations $n_{\mathbf{k}_{1}}$ can be used as dynamical variables ${ }^{36}$ which helps to understand the dynamics of the physical variables and simplifies the equations of motion.

$$
\begin{aligned}
& \langle\mathbf{S}(z)\rangle=\sum_{n n^{\prime}} \mathbf{S}_{n n^{\prime}} M_{n}^{n^{\prime}}(z), \\
& n_{\mathbf{k}_{1}}=\sum_{\sigma} C_{\sigma \mathbf{k}_{1}}^{\sigma}, \\
& \mathbf{s}_{\mathbf{k}_{1}}=\sum_{\sigma_{1} \sigma_{2}} \mathbf{s}_{\sigma_{1} \sigma_{2}} C_{\sigma_{1} \mathbf{k}_{1}}^{\sigma_{2}}, \\
& Q_{j \mathbf{k}_{1}}^{\alpha \mathbf{k}_{2}}:=\sum_{\substack{\sigma_{1} \sigma_{2} \\
n_{1} n_{2}}} S_{n_{1} n_{2}}^{j} s_{\sigma_{1} \sigma_{2}}^{\alpha} Q_{\sigma_{1} n_{1} \mathbf{k}_{1}}^{\sigma_{2} n_{2} \mathbf{k}_{2}} .
\end{aligned}
$$

From now on, we will use the convention that $\sigma$-indices describe spin-up and spin-down subbands, $n$-indices enumerate the impurity states, while all other Latin indices represent three-dimensional geometric directions, e.g., $j \in\{1,2,3\}$, and Greek indices range from 0 to 3 , where the 0 describes occupations. In this notation, 
the zeroth spin matrix is defined to be the $2 \times 2$ identity matrix $s_{\sigma_{1} \sigma_{2}}^{0}=\delta_{\sigma_{1} \sigma_{2}}$. Furthermore, we adopt the Einstein notation, so that when the same index appears twice, a summation is implied. Sub- and superscripts are used, e.g., to distinguish the carrier and impurity degrees of freedom of the correlations, and do not represent a covariant formulation. Sums over $\mathbf{k}$ vectors, on the other hand, will be stated explicitly and no sum is implied, if an index $\mathbf{k}_{i}$ appears twice in an expression.

In this notation, the equations of motion of Ref. 36 and 37, extended by terms due to the $\mathbf{k}$-dependent effective magnetic field and the impurity and carrier Zeeman energies, are:

$$
\begin{aligned}
& \frac{\partial}{\partial t}\left\langle S^{l}(z)\right\rangle=\left(\omega_{M n}(z) \times\langle\mathbf{S}(z)\rangle\right)_{l}-\frac{J_{s d}|\psi(z)|^{2} d}{\hbar V^{2}} \sum_{\mathbf{k} \mathbf{k}^{\prime}} \epsilon_{i j l} \operatorname{Re}\left(Q_{i \mathbf{k}}^{j \mathbf{k}^{\prime}}(z)\right) \\
& \frac{\partial}{\partial t} n_{\mathbf{k}_{1}}=\int_{-\frac{d}{2}}^{\frac{d}{2}} d z \frac{J_{s d}|\psi(z)|^{2} N_{M n}}{\hbar V^{2}} \sum_{\mathbf{k}} 2 \operatorname{Im}\left(Q_{i \mathbf{k}_{1}}^{i \mathbf{k}}(z)\right) \\
& \frac{\partial}{\partial t} s_{\mathbf{k}_{1}}^{l}=\left(\boldsymbol{\Omega}_{\mathbf{k}_{1}}^{\prime} \times \mathbf{s}_{\mathbf{k}_{1}}\right)_{l}+\int_{-\frac{d}{2}}^{\frac{d}{2}} d z \frac{J_{s d}|\psi(z)|^{2} N_{M n}}{\hbar V^{2}} \sum_{\mathbf{k}} \operatorname{Im}\left[\frac{1}{2} Q_{l \mathbf{k}_{1}}^{0 \mathbf{k}}(z)+i \epsilon_{i j l} Q_{i \mathbf{k}_{1}}^{j \mathbf{k}}(z)\right] \\
& \frac{\partial}{\partial t} Q_{l \mathbf{k}_{1}}^{\alpha \mathbf{k}_{2}}(z)=-i\left(\omega_{\mathbf{k}_{2}}-\omega_{\mathbf{k}_{1}}\right) Q_{l \mathbf{k}_{1}}^{\alpha \mathbf{k}_{2}}(z)+\left(A_{\mathbf{k}_{1}}+A_{\mathbf{k}_{2}}^{*}\right) \\
& b_{l \mathbf{k}_{1}}^{\alpha \mathbf{k}_{2}}(z)=\frac{i}{\hbar} J_{s d}^{\gamma} d \mid \psi(z) \mathbf{k}_{1} \\
& \left.\left.\mathbf{k}_{1}(z)+\epsilon_{i j l} \omega_{M n}^{i}(z) Q_{j \mathbf{k}_{1}}^{\alpha}(z)\right\rangle\left\langle s^{i} s^{\alpha}\right\rangle_{\mathbf{k}_{2}} \mathbf{k}_{1}-\left\langle S^{l} S^{i}(z)\right\rangle\left\langle s^{\alpha} s^{i}\right\rangle_{\mathbf{k}_{1}} \mathbf{k}_{2}\right]
\end{aligned}
$$

where the mean-field precession frequencies for impurities and carriers are defined as

$$
\begin{aligned}
& \boldsymbol{\omega}_{M n}(z):=\frac{g_{M n} \mu_{B}}{\hbar} \mathbf{B}+\frac{J_{s d}|\psi(z)|^{2} d}{\hbar V} \sum_{\mathbf{k}} \mathbf{s}_{\mathbf{k}} \\
& \boldsymbol{\Omega}_{\mathbf{k}}^{\prime}:=\boldsymbol{\Omega}_{\mathbf{k}}+\boldsymbol{\omega}_{e}(\mathbf{k}) \\
& \boldsymbol{\omega}_{e}(\mathbf{k}):=\frac{g_{e}(\mathbf{k}) \mu_{B}}{\hbar} \mathbf{B}+\int_{-\frac{d}{2}}^{\frac{d}{2}} d z \frac{J_{s d}|\psi(z)|^{2} N_{M n}}{\hbar V}\langle\mathbf{S}(z)\rangle,
\end{aligned}
$$

The k-dependent precession-like movement of the electron degree of freedom of the correlations is described by the $4 \times 4$ matrix

$$
A_{\mathbf{k}_{1}}:=\left(\begin{array}{cc}
0 & \left(i \boldsymbol{\Omega}_{\mathbf{k}_{1}}^{\prime}\right)^{T} \\
\left(\frac{i}{4} \boldsymbol{\Omega}_{\mathbf{k}_{1}}^{\prime}\right) & \frac{1}{2}\left[\boldsymbol{\Omega}_{\mathbf{k}_{\mathbf{1}}}^{\prime}\right]_{\times}
\end{array}\right),
$$

where $\left[\boldsymbol{\Omega}_{\mathbf{k}_{1}}^{\prime}\right]_{\times}$is the $3 \times 3$ cross-product matrix with $\left[\boldsymbol{\Omega}_{\mathbf{k}_{1}}^{\prime}\right]_{\times} \mathbf{v}=\boldsymbol{\Omega}_{\mathbf{k}_{1}}^{\prime} \times \mathbf{v}$.

The source terms $b_{l \mathbf{k}_{1}}^{\alpha \mathbf{k}_{2}}(z)$ involve electron variables $n_{\mathbf{k}}$ and $\mathbf{s}_{\mathbf{k}}$ in the form:

$$
\begin{gathered}
\left\langle s^{i} s^{j}\right\rangle_{\mathbf{k}_{1}}^{\mathbf{k}_{2}}:=\delta_{i j}\left[\frac{1}{4}\left(1-\frac{n_{\mathbf{k}_{2}}}{2}\right) n_{\mathbf{k}_{1}}+\frac{1}{2} \mathbf{S}_{\mathbf{k}_{1}} \cdot \mathbf{S}_{\mathbf{k}_{2}}\right]-\frac{1}{2} s_{\mathbf{k}_{1}}^{i} s_{\mathbf{k}_{2}}^{j}+ \\
-\frac{1}{2} s_{\mathbf{k}_{1}}^{j} s_{\mathbf{k}_{2}}^{i}+\frac{i}{2} \epsilon_{i j l}\left[\left(1-\frac{n_{\mathbf{k}_{2}}}{2}\right) s_{\mathbf{k}_{1}}^{l}+\frac{n_{\mathbf{k}_{1}}}{2} s_{\mathbf{k}_{2}}^{l}\right]
\end{gathered}
$$

and

$$
\begin{aligned}
\left\langle s^{i} s^{0}\right\rangle_{\mathbf{k}_{2}}^{\mathbf{k}_{1}}:=\left(1-\frac{n_{\mathbf{k}_{1}}}{2}\right) s_{\mathbf{k}_{2}}^{i}-\frac{n_{\mathbf{k}_{2}}}{2} s_{\mathbf{k}_{1}}^{i}-i \epsilon_{i j l} s_{\mathbf{k}_{1}}^{j} s_{\mathbf{k}_{2}}^{l} \\
\left\langle s^{0} s^{i}\right\rangle_{\mathbf{k}_{1}}^{\mathbf{k}_{2}}:=\left(1-\frac{n_{\mathbf{k}_{2}}}{2}\right) s_{\mathbf{k}_{1}}^{i}-\frac{n_{\mathbf{k}_{1}}}{2} s_{\mathbf{k}_{2}}^{i}-i \epsilon_{i j l} s_{\mathbf{k}_{1}}^{j} s_{\mathbf{k}_{2}}^{l} .
\end{aligned}
$$

Also, $b_{l \mathbf{k}_{1}}^{\alpha \mathbf{k}_{2}}(z)$ contains second moments of the impurity variables:

$$
\begin{aligned}
& \left\langle S^{i} S^{j}(z)\right\rangle=\left\langle S^{\perp^{2}}(z)\right\rangle \delta_{i j}+\left\langle S^{\|^{2}}(z)-S^{\perp^{2}}(z)\right\rangle \times \\
& \frac{\left\langle S^{i}(z)\right\rangle\left\langle S^{j}(z)\right\rangle}{\langle\mathbf{S}(z)\rangle^{2}}+\frac{i}{2} \epsilon_{i j l}\left\langle S^{l}(z)\right\rangle,
\end{aligned}
$$

where $S^{\|}:=\frac{\mathbf{S} \cdot\langle\mathbf{S}\rangle}{\langle\mathbf{S}\rangle^{2}}$ is the spin operator projected onto the direction of the average impurity spin and $\left\langle S^{\perp^{2}}\right\rangle=$ $\frac{1}{2}\left\langle S^{2}-S^{\|^{2}}\right\rangle$ is the perpendicular second moment, with $\left\langle S^{2}\right\rangle=\frac{S(S+1)}{4}=\frac{35}{4}$ for a spin- $\frac{5}{2}$ system.

By going over from the density matrices in Eqs. (8) as dynamical variables to the variables defined in Eqs. (9), one ends up with a set of equations that is not closed. Thus, some approximations have to be employed to evaluate the right-hand side of Eqs. (10): First of all, it is necessary to evaluate the second moments of the impurity magnetization, for which the equations of motion can in principle be calculated, but they involve even higher moments. We reduce the complexity of the equations by calculating a quasi-thermal impurity density matrix in 
each time step, which is consistent with the average spin $\langle\mathbf{S}(z)\rangle$. Furthermore, the source terms $c_{l \mathbf{k}_{1}}^{\alpha \mathbf{k}_{2}}(z)^{45}$ contain degrees of freedom of the original correlation functions $Q_{\sigma_{1} n_{1} \mathbf{k}_{1}}^{\sigma_{2} n_{2} \mathbf{k}_{2}}$ that are not expressible in terms of $Q_{l \mathbf{k}_{1}}^{\alpha \mathbf{k}_{2}}$. However, the terms $c_{l \mathbf{k}_{1}}^{\alpha \mathbf{k}_{2}}(z)$ were shown to be irrelevant in numerical calculations in the situation described in Ref. 37. Since these terms are proportional to some correlation functions $Q_{\sigma n \mathbf{k}}^{\sigma^{\prime} n^{\prime} \mathbf{k}^{\prime}}$, they mainly renormalize the frequencies with which the correlations oscillate. As will be seen later, the values of these frequencies determine the difference in kinetic energies of the initial and final states of carriers scattered due to the $s$ - $d$ interaction. On the other hand it will be shown that neglecting the terms $c_{l \mathbf{k}_{1}}^{\alpha \mathbf{k}_{2}}(z)$ leads to equations that conserve the mean-field energies of the carriers, so that the role of these terms is mainly to ensure energy conservation including the carrier-impurity correlation energy. However, this correlation energy is typically of the order of a few $\mu \mathrm{eV}^{28}$, so that it is typically a good approximation to neglect the source terms $c_{l \mathbf{k}_{1}}^{\alpha \mathbf{k}_{2}}(z)$, as we will henceforth do.

With these approximations, it seems straightforward to solve the coupled system of ordinary differential equations (10) numerically. However, this task is very challenging, since the correlations are indexed by two $\mathbf{k}$-vectors, where each one is an element of a twodimensional continuum in the case of a quantum well. The problem therefore has the complexity $\mathcal{O}\left(N_{k}^{4} N_{z} N_{t}\right)$, where $N_{k}, N_{z}$ and $N_{t}$ are the numbers of discretization points of the k-space (linear dimension), the growthdirection in real space and the time, respectively. Our strategy to make the calculation tractable follows Ref. 37: The computation time can be strongly reduced, if the correlations are eliminated and only their effects on the electron and impurity variables are kept. This can be achieved by formally integrating the equations of motion for the correlations at the cost of introducing a memory integral. This memory integral can in turn be eliminated by a short-memory or Markov approximation, which is established in the next section.

\section{Derivation and Applicability of the Markov limit}

Before we discuss the Markov limit of the correlations including the precession-like movement of the correlations, we briefly recapitulate the standard way ${ }^{36,46}$ of deriving the Markov limit of quantum kinetic equations in the simplest possible situation with $\boldsymbol{\Omega}_{\mathbf{k}}^{\prime}=0$ and $\boldsymbol{\omega}_{M n}(z)=0$. There, the equation of motion (10d) for the correlations becomes

$$
\frac{\partial}{\partial t} Q_{l \mathbf{k}_{1}}^{\alpha \mathbf{k}_{2}}=-i\left(\omega_{\mathbf{k}_{2}}-\omega_{\mathbf{k}_{1}}\right) Q_{l \mathbf{k}_{1}}^{\alpha \mathbf{k}_{2}}+b_{l \mathbf{k}_{1}}^{\alpha \mathbf{k}_{2}}
$$

If the source term $b_{l \mathbf{k}_{1}}^{\alpha \mathbf{k}_{2}}$ is regarded as a time-dependent inhomogeneity, one can first solve the homogeneous part of the equation and take the inhomogeneity into account by a variation of constants, which yields:

$$
\begin{aligned}
Q_{l \mathbf{k}_{1}}^{\alpha \mathbf{k}_{2}}(t)= & e^{-i\left(\omega_{\mathbf{k}_{2}}-\omega_{\mathbf{k}_{1}}\right) t}\left[Q_{l \mathbf{k}_{1}}^{\alpha \mathbf{k}_{2}}\left(t_{0}\right)+\right. \\
& \left.+\int_{t_{0}}^{t} d t^{\prime} e^{i\left(\omega_{\mathbf{k}_{2}}-\omega_{\mathbf{k}_{1}}\right) t^{\prime}} b_{l \mathbf{k}_{1}}^{\alpha \mathbf{k}_{2}}\left(t^{\prime}\right)\right] .
\end{aligned}
$$

We assume that the carriers stem exclusively form optical excitation and therefore also the correlations are zero before the laser pulse is applied. Therefore, $Q_{l \mathbf{k}_{1}}^{\alpha \mathbf{k}_{2}}\left(t_{0}\right)=0$ for $t_{0} \rightarrow-\infty$. The correlations act back on the carrier and impurity variables only via sums over correlations with respect to at least one $\mathbf{k}$-index. Thus, we consider, e.g.,

$$
\sum_{\mathbf{k}_{2}} Q_{l \mathbf{k}_{1}}^{\alpha \mathbf{k}_{2}}(t)=\int_{0}^{\omega_{B Z}} d \omega D(\omega) \int_{-\infty}^{t} d t^{\prime} e^{i\left(\omega-\omega_{\mathbf{k}_{1}}\right)\left(t^{\prime}-t\right)} b_{l \mathbf{k}_{1}}^{\alpha \mathbf{k}(\omega)}\left(t^{\prime}\right),
$$

with the quasi-continuous limit

$$
\sum_{\mathbf{k}} \cdots \rightarrow \int_{B Z} d k D(k) \cdots=\int_{0}^{\omega_{B} Z} d \omega D(\omega) \ldots,
$$

where $\hbar \omega$ are the spin-independent single-particle energies of $H_{0}$ and $\hbar \omega_{B Z}$ is a cut-off energy corresponding to the upper end of the conduction band. Although this expression is valid also for non-parabolic band structures, we simplify the discussion by first assuming an effective mass approximation in two dimensions, so that $D^{2 D}:=D(\omega)=\frac{A m^{*}}{2 \pi \hbar}$ is constant.

Now, the Markov or short-memory approximation can be applied to Eq. (16) as follows: Assuming that, because of the $\mathbf{k}$-sum, the effects of the correlations on the carrier and impurity dynamics dephase fast for not too small values of $t^{\prime}-t$ in the integral kernel, the largest contribution of the integrals stems from source terms $b_{l \mathbf{k}_{1}}^{\alpha \mathbf{k}(\omega)}\left(t^{\prime}\right)$ with $t^{\prime} \approx t$. Then, Eq. (16) can be approximated by

$$
\sum_{\mathbf{k}_{2}} Q_{l \mathbf{k}_{1}}^{\alpha \mathbf{k}_{2}}(t) \approx D^{2 D} \int_{0}^{\omega_{B Z}} d \omega b_{l \mathbf{k}_{1}}^{\alpha \mathbf{k}(\omega)}(t) \int_{-\infty}^{t} d t^{\prime} e^{i\left(\omega-\omega_{\mathbf{k}_{1}}\right)\left(t^{\prime}-t\right)}
$$

Using the Sokhotski-Plemelj formula

$$
\int_{-\infty}^{0} d t^{\prime} e^{i x t^{\prime}}=\pi\left(\delta(x)-\mathcal{P} \frac{i}{\pi x}\right)=: \pi \bar{\delta}(x),
$$

where $\mathcal{P}$ denotes the Cauchy principal value, allows to express the correlations solely in terms of carrier and impurity variables evaluated at $t^{\prime}=t$. For the real part of $\bar{\delta}$, the $\mathbf{k}$-sum reduces to an integration over a single energy shell. The imaginary part has been shown to lead to a small renormalization of the precession frequencies ${ }^{28}$ 
that can only reach values over $1 \%$ for a small range of realistic material parameters and excitation conditions, so that we consider only the real part of $\bar{\delta}$ in the further discussion of the Markov limit.

In the above treatment, it was postulated that the memory induced by the correlations is short. To see in which cases this is indeed a good approximation and how the timescale of the memory can be defined, we briefly summarize the findings of Ref. 47: The source terms $b_{l \mathbf{k}_{1}}^{\alpha \mathbf{k}_{2}}$ that enter, e.g., in the dynamics for the carrier spin $\mathbf{s}_{\mathbf{k}_{1}}$, involve the variables $n_{\mathbf{k}_{1}}, \mathbf{s}_{\mathbf{k}_{1}}, n_{\mathbf{k}_{2}}$ and $\mathbf{s}_{\mathbf{k}_{2}}$. For the parts that only contain variables at $\mathbf{k}_{1}$, which we will refer to as $b_{l \mathbf{k}_{1}}^{\alpha}$, the real part of the integral on the right-hand side of Eq. (16) yields:

$$
\begin{aligned}
& \operatorname{Re} \int_{-\infty}^{t} d t^{\prime} \int_{0}^{\omega_{B Z}} d \omega e^{i\left(\omega-\omega_{\mathbf{k}_{1}}\right)\left(t^{\prime}-t\right)} b_{l \mathbf{k}_{1}}^{\alpha}\left(t^{\prime}\right)= \\
& =\operatorname{Re} \int_{-\infty}^{0} d t^{\prime \prime} \frac{\sin \left[\left(\omega_{B Z}-\omega_{\mathbf{k}_{1}}\right) t^{\prime \prime}\right]+\sin \left(\omega_{\mathbf{k}_{1}} t^{\prime \prime}\right)}{t^{\prime \prime}} b_{l \mathbf{k}_{1}}^{\alpha}\left(t+t^{\prime \prime}\right)
\end{aligned}
$$

Since $\frac{\sin \Delta \omega t}{t} \rightarrow \pi \delta(t)$ for $\Delta \omega \rightarrow \infty$, this way of expressing the integral now shows that the memory has two timescales, one corresponding to $\left(\omega_{B Z}-\omega_{\mathbf{k}_{1}}\right)^{-1}$, which is typically of the order of a few fs due to values of $\omega_{B Z}$ in the $\mathrm{eV}$ range, and the other one at $\omega_{\mathbf{k}_{1}}^{-1}$. This can explain, why for a $\delta$-like initial electron occupation at $\mathbf{k}_{1}=0$, the spin transfer rate extracted from the quantum kinetic calculations in Ref. 47 is exactly $\frac{1}{2}$ of the Markovian expression for the rate. Thus, non-Markovian effects are found to be mainly due to the spectral proximity of the electrons to the band edge. Therefore, if the initial carrier distribution has a width of a few $\mathrm{meV}$, the Markovian results coincide with the quantum kinetic calculations ${ }^{47}$.

For the other parts of the source terms $b_{l \mathbf{k}_{1}}^{\alpha \mathbf{k}_{2}}$ which depend also on the electron variables at $\mathbf{k}_{2}$, a new timescale emerges which corresponds to the inverse of the frequency difference $\tau_{\mathbf{k}_{1}, \mathbf{k}_{2}}$ for which the electron variables $\mathbf{S}_{\mathbf{k}_{2}}\left(n_{\mathbf{k}_{2}}\right)$ start to differ notably from $\mathbf{s}_{\mathbf{k}_{1}}\left(n_{\mathbf{k}_{1}}\right)$.

In summary, it can therefore be said that the correlation time $\tau_{c o r}$, i. e. the timescale of the memory induced by the correlations, depends on the details of the spectral carrier distributions. Thus, in order to obtain meaningful results by using the Markov approximation, it is of key importance that the dynamics of the source terms takes place on a much slower timescale than the build-up of correlations $\tau_{c o r}$. If this is not the case, e.g., due to a fast precession of the electron spins with a frequency $\omega_{e}$, it is necessary to split this precession off of the correlation induced spin transfer, yielding a modified integral kernel $e^{i\left(\omega_{\mathbf{k}_{2}}-\omega_{\mathbf{k}_{1}} \pm \omega_{e}\right) t^{\prime}}$ and therefore a shift of $\pm \omega_{e}$ in the respective $\delta$-functions ${ }^{37}$. Therefore, the identification of fast and slowly changing parts of the source terms $b_{l \mathbf{k}_{1}}^{\alpha \mathbf{k}_{2}}$ is crucial for the derivation of the Markov limit of the quantum kinetic equations of motion (10).

\section{Markov Limit of the Quantum Kinetic Equations}

In the last section, the standard procedure of deriving a Markov limit was summarized starting from a simple set of equations where all the relevant spin precessions in DMS were neglected. Now, for the more general theory of the present article, we repeat the same steps while accounting for all terms in Eqs. (10). As above, first of all, the homogeneous part of the differential equation for the correlations has to be solved.

$$
\begin{aligned}
& \frac{\partial}{\partial t} Q_{l \mathbf{k}_{1}}^{\alpha \mathbf{k}_{2}}=-i\left(\omega_{\mathbf{k}_{2}}-\omega_{\mathbf{k}_{1}}\right) Q_{l \mathbf{k}_{1}}^{\alpha \mathbf{k}_{2}}+ \\
& +\left(A_{\mathbf{k}_{1}}+A_{\mathbf{k}_{2}}^{*}\right)_{\alpha \gamma} Q_{l \mathbf{k}_{1}}^{\gamma \mathbf{k}_{2} \text { hom }}+\epsilon_{i j l} \omega_{M n}^{i} Q_{j \mathbf{k}_{1}}^{\alpha \mathbf{k}_{2} \text { hom }} .
\end{aligned}
$$

Eq. (21) can be represented in a more abstract form, if $Q_{l \mathbf{k}_{1}}^{\alpha \mathbf{k}_{2}}$ hom is rewritten as a single vector $\mathbf{Q}^{\text {hom }}$ with respect to the set of indices $l, \alpha, \mathbf{k}_{1}$ and $\mathbf{k}_{2}$. Then, Eq. (21) becomes:

$$
\frac{\partial}{\partial t} \mathbf{Q}^{\text {hom }}=M \mathbf{Q}^{\text {hom }}
$$

where the matrix $M$ is defined by the terms on the r. h. s. of Eq. (21). The formal solution of Eq. (22) is the time ordered exponential:

$$
\mathbf{Q}^{\mathrm{hom}}\left(t_{0}+\Delta t\right)=\mathcal{T} e^{\int_{t_{0}}^{t_{0}+\Delta t} d t^{\prime} M\left(t^{\prime}\right)} \mathbf{Q}^{\mathrm{hom}}\left(t_{0}\right)
$$

However, since in the Markov limit the solution of the homogeneous differential equation is only required on a timescale comparable to $\tau_{\text {cor }}$ in the fs range, we can assume that neither the precession frequencies nor the precession axes will change significantly on this timescale. This assumption makes it possible to approximate $M\left(t^{\prime}\right) \approx M\left(t_{0}\right)$ in Eq. (23) so that the time ordering operator $\mathcal{T}$ can be dropped.

The expression for the solution for $\mathbf{Q}^{\text {hom }}$ can be further simplified, because the different contributions to the r. h. s. of Eq. (21) act on different degrees of freedom of $Q_{l \mathbf{k}_{1}}^{\alpha \mathbf{k}_{2}}$ hom and therefore commute. As also $A_{\mathbf{k}_{1}}$ and $A_{\mathbf{k}_{2}}^{*}$ commute, which can be checked directly using the explicit expression for those matrices in Eq. (11d), the homogeneous part of the equation of motion for the correlation is solved by

$$
\begin{aligned}
& Q_{l \mathbf{k}_{1}}^{\alpha \mathbf{k}_{2} \text { hom }}\left(t_{0}+\Delta t\right)=e^{-i\left(\omega_{\mathbf{k}_{2}}-\omega_{\mathbf{k}_{1}}\right) \Delta t} \times \\
& \left(e^{A_{\mathbf{k}_{1}} \Delta t} e^{A_{\mathbf{k}_{2}}^{*} \Delta t}\right)_{\alpha \gamma}\left(e^{\left[\boldsymbol{\omega}_{M n}\right]_{\times} \Delta t}\right)_{l l^{\prime}} Q_{l \mathbf{k}_{1}}^{\alpha \mathbf{k}_{2}}{ }^{h o m}\left(t_{0}\right)
\end{aligned}
$$

The exponential $e^{\left[\boldsymbol{\omega}_{M n}\right]_{\times} t}$ of the cross product matrix $\left[\boldsymbol{\omega}_{M n}\right]_{\times}$is

$$
e^{\left[\boldsymbol{\omega}_{M n}\right] \times t}=R_{\boldsymbol{\omega}_{M n}}\left(\omega_{M n} t\right),
$$


where $R_{\mathbf{n}}(\alpha)$ is the $3 \times 3$ matrix describing a rotation around the axis $\mathbf{n}$ with an angle $\alpha$. Similarly, it is possible to calculate an exponential of the matrices $A_{\mathrm{k}}$ :

$$
\begin{aligned}
& E_{\mathbf{k}_{1}}(t):=e^{A \mathbf{k}_{1} t}= \\
& =\cos \left(\frac{\Omega_{\mathbf{k}_{1}}^{\prime}}{2} t\right) \mathbf{1}+\sin \left(\frac{\Omega_{\mathbf{k}_{1}}^{\prime}}{2} t\right)\left(\begin{array}{cc}
0 & 2 i \frac{\boldsymbol{\Omega}_{\mathbf{k}_{1}}^{\prime T}}{\Omega_{\mathbf{k}_{1}}} \\
\frac{i}{2} \frac{\Omega_{\mathbf{k}_{1}}^{\prime}}{\Omega_{\mathbf{k}_{1}}} & {\left[\frac{\Omega_{\mathbf{k}_{1}}^{\prime}}{\Omega_{\mathbf{k}_{1}}}\right]_{\times}}
\end{array}\right),
\end{aligned}
$$

with the inverse $\left(E_{\mathbf{k}_{1}}(t)\right)^{-1}=E_{\mathbf{k}_{1}}(-t)$.

Now, the solution to the inhomogeneous equation can be found by a variation of constants yielding:

$$
\begin{aligned}
& Q_{l \mathbf{k}_{1}}^{\alpha \mathbf{k}_{2}}\left(t_{0}+\Delta t\right)=e^{-i\left(\omega_{\mathbf{k}_{2}}-\omega_{\mathbf{k}_{1}}\right) \Delta t}\left(E_{\mathbf{k}_{1}}(\Delta t) E_{\mathbf{k}_{2}}^{*}(\Delta t)\right)_{\alpha \gamma} \times \\
& \left(R_{\boldsymbol{\omega}_{M n}}\left(\omega_{M n} \Delta t\right)\right)_{l l^{\prime}}\left[Q_{l \mathbf{k}_{1}}^{\alpha \mathbf{k}_{2}}\left(t_{0}\right)+\int_{t_{0}}^{t_{0}+\Delta t} d t^{\prime} e^{i\left(\omega_{\mathbf{k}_{2}}-\omega_{\mathbf{k}_{1}}\right) t^{\prime}} \times\right. \\
& \left.\left(E_{\mathbf{k}_{1}}\left(-t^{\prime}\right) E_{\mathbf{k}_{2}}^{*}\left(-t^{\prime}\right)\right)_{\gamma \kappa}\left(R_{\boldsymbol{\omega}_{M n}}\left(-\omega_{M n} t^{\prime}\right)\right)_{l^{\prime} l^{\prime \prime}} b_{l^{\prime \prime} \mathbf{k}_{1}}^{\kappa \mathbf{k}_{2}}\left(t^{\prime}\right)\right]
\end{aligned}
$$

Eq. (27) can be further simplified by decomposing the matrices $R_{\boldsymbol{\omega}_{M n}}\left(\omega_{M n} t\right)$ and $E_{\mathbf{k}_{1}}$ as well as $E_{\mathbf{k}_{2}}^{*}$ in components oscillating with different frequencies:

$$
\begin{aligned}
& R_{\mathbf{n}}(\omega t)=R_{\mathbf{n}}^{0}+R_{\mathbf{n}}^{+} e^{i \omega t}+R_{\mathbf{n}}^{-} e^{-i \omega t} \\
& E_{\mathbf{k}_{1}}(t)=E_{\mathbf{k}_{1}}^{0}+E_{\mathbf{k}_{1}}^{+} e^{i \frac{1}{2} \Omega_{\mathbf{k}_{1}}^{\prime} t}+E_{\mathbf{k}_{1}}^{-} e^{-i \frac{1}{2} \Omega_{\mathbf{k}_{1}}^{\prime} t} \\
& E_{\mathbf{k}_{2}}^{*}(t)=\left(E_{\mathbf{k}_{2}}^{*}\right)^{0}+\left(E_{\mathbf{k}_{2}}^{*}\right)^{+} e^{i \frac{1}{2} \Omega_{\mathbf{k}_{2}}^{\prime} t}+\left(E_{\mathbf{k}_{2}}^{*}\right)^{-} e^{-i \frac{1}{2} \Omega_{\mathbf{k}_{2}}^{\prime} t}
\end{aligned}
$$

where the components of $E_{\mathbf{k}}$ can directly be read off from the definition in Eq. (26) and the decomposition of $R_{\mathbf{n}}(\alpha)$ is

$$
\begin{aligned}
& \left(R_{\mathbf{n}}^{0}\right)_{i j}=\frac{n_{i} n_{j}}{|\mathbf{n}|^{2}} \\
& \left(R_{\mathbf{n}}^{ \pm}\right)_{i j}=\frac{1}{2}\left(\delta_{i j}-\frac{n_{i} n_{j}}{|\mathbf{n}|^{2}} \pm i \epsilon_{i j k} \frac{n_{k}}{|\mathbf{n}|}\right) .
\end{aligned}
$$

For the components defined in Eq. (28), an important relation is

$$
\begin{aligned}
& R_{\mathbf{n}}^{\chi_{1}} R_{\mathbf{n}}^{\chi_{2}}=\delta_{\chi_{1} \chi_{2}} R_{\mathbf{n}}^{\chi_{1}}, \\
& E_{\mathbf{k}_{1}}^{\chi_{1}} E_{\mathbf{k}_{1}}^{\chi_{2}}=\delta_{\chi_{1} \chi_{2}} E_{\mathbf{k}_{1}}^{\chi_{1}}, \\
& \left(E_{\mathbf{k}_{2}}^{*}\right)^{\chi_{1}}\left(E_{\mathbf{k}_{2}}^{*}\right)^{\chi_{2}}=\delta_{\chi_{1} \chi_{2}}\left(E_{\mathbf{k}_{2}}^{*}\right)^{\chi_{1}},
\end{aligned}
$$

where from now on we assume $\chi_{i} \in\{-1,0,1\}$ for any $\chi$-index.

As stated earlier, it is necessary to identify fast oscillating and slowly changing contributions to the source terms $b_{l \mathbf{k}_{1}}^{\alpha \mathbf{k}_{2}}$. To this end, we consider the dynamics of $b_{l \mathbf{k}_{1}}^{\alpha \mathbf{k}_{2}}$ in the mean-field approximation, where

$$
\begin{aligned}
\left\langle S^{i} S^{j}\left(t_{0}+\Delta t\right)\right\rangle \approx & \left(R_{\boldsymbol{\omega}_{M n}}\left(\omega_{M n} \Delta t\right)\right)_{i i^{\prime}} \times \\
& \left(R_{\boldsymbol{\omega}_{M n}}\left(\omega_{M n} \Delta t\right)\right)_{j j^{\prime}}\left\langle S^{i^{\prime}} S^{j^{\prime}}\left(t_{0}\right)\right\rangle \\
n_{\mathbf{k}}\left(t_{0}+\Delta t\right) \approx & n_{\mathbf{k}}\left(t_{0}\right) \\
s_{\mathbf{k}}^{i}\left(t_{0}+\Delta t\right) \approx & \left(R_{\boldsymbol{\Omega}_{\mathbf{k}}^{\prime}}\left(\Omega_{\mathbf{k}}^{\prime} \Delta t\right)\right)_{i i^{\prime}} s_{\mathbf{k}}^{i^{\prime}}\left(t_{0}\right)
\end{aligned}
$$

With these approximations, the source terms can be decomposed into

$$
b_{l \mathbf{k}_{1}}^{\alpha \mathbf{k}_{2}}\left(t_{0}+\Delta t\right) \approx \sum_{m} b_{l \mathbf{k}_{1}}^{\alpha \mathbf{k}_{2}\left(\omega_{m}\right)}\left(t_{0}\right) e^{i \omega_{m} \Delta t},
$$

where $m$ counts all the possible oscillation frequencies $\omega_{m}$ which consist of combinations of the frequencies $\omega_{M n}(z)$ and $\Omega_{\mathbf{k}}^{\prime}$.

Now, the Markov limit of the Eqs. (10) can be established by using the Markov approximation in Eq. (18) with the Sokhotski-Plemelj formula in Eq. (19) on the expression for the time evolution of the correlations in Eq. (27), simplifying the products of exponential matrices with the relations (30) and decomposing the source terms according to Eq. (32):

$$
\begin{aligned}
& Q_{l \mathbf{k}_{1}}^{\alpha \mathbf{k}_{2}} \approx \pi \sum_{m} \sum_{\chi_{M n}, \chi_{\mathbf{k}_{1}}, \chi_{\mathbf{k}_{2}}} \bar{\delta}\left(\omega_{\mathbf{k}_{2}}-\left(\omega_{\mathbf{k}_{1}}+\chi_{M n} \omega_{M n}+\right.\right. \\
& \left.\left.+\frac{1}{2} \chi_{\mathbf{k}_{1}} \Omega_{\mathbf{k}_{1}}^{\prime}+\frac{1}{2} \chi_{\mathbf{k}_{2}} \Omega_{\mathbf{k}_{2}}^{\prime}-\omega_{m}\right)\right) \times \\
& \left(E_{\mathbf{k}_{1}}^{\chi_{\mathbf{k}_{1}}}\left(E_{\mathbf{k}_{2}}^{*}\right)^{\chi_{\mathbf{k}_{2}}}\right)_{\alpha \gamma}\left(R_{\omega_{M n}}^{\chi_{M n n}}\right)_{l l^{\prime}} b_{l^{\prime} \mathbf{k}_{1}}^{\gamma \mathbf{k}_{2}\left(\omega_{m}\right)}\left(t^{\prime}\right)
\end{aligned}
$$

or more explicitly: 


$$
\begin{aligned}
& Q_{l \mathbf{k}_{1}}^{\alpha \mathbf{k}_{2}}(z) \approx \pi \frac{i}{\hbar} J_{s d}|\psi(z)|^{2} d \sum_{\chi_{\mathbf{k}_{1}}, \chi_{\mathbf{k}_{1}}^{\prime}, \chi_{\mathbf{k}_{2}}, \chi_{\mathbf{k}_{2}}^{\prime}, \chi_{M n}} \bar{\delta}\left(\omega_{\mathbf{k}_{2}}-\left(\omega_{\mathbf{k}_{1}}+\left(\frac{1}{2} \chi_{\mathbf{k}_{1}}-\chi_{\mathbf{k}_{1}}^{\prime}\right) \Omega_{\mathbf{k}_{1}}^{\prime}+\left(\frac{1}{2} \chi_{\mathbf{k}_{2}}-\chi_{\mathbf{k}_{2}}^{\prime}\right) \Omega_{\mathbf{k}_{2}}^{\prime}-\chi_{M n} \omega_{M n}(z)\right)\right)\{
\end{aligned}
$$

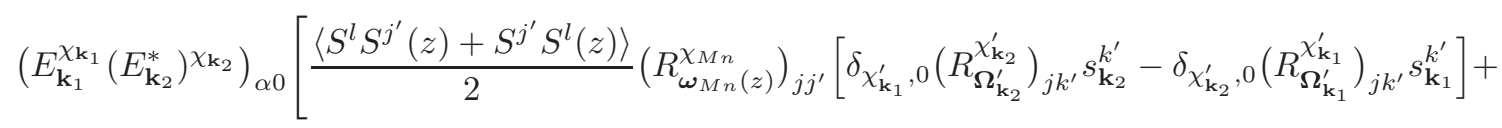

$$
\begin{aligned}
& \left.+\frac{i}{2} \epsilon_{j^{\prime} l i^{\prime \prime}}\left\langle S^{i^{\prime \prime}}(z)\right\rangle\left(R_{\boldsymbol{\omega}_{M n}(z)}^{\chi_{M n}}\right)_{j j^{\prime}}\left[\delta_{\chi_{\mathbf{k}_{1}}^{\prime}, 0}\left(1-n_{\mathbf{k}_{1}}\right)\left(R_{\boldsymbol{\Omega}_{\mathbf{k}_{2}}^{\prime}}^{\chi_{\mathbf{k}_{2}}^{\prime}}\right)_{j k^{\prime}} s_{\mathbf{k}_{2}}^{k^{\prime}}+\delta_{\chi_{\mathbf{k}_{2}}^{\prime}, 0}\left(1-n_{\mathbf{k}_{2}}\right)\left(R_{\mathbf{\Omega}_{\mathbf{k}_{1}}^{\prime}}^{\chi_{\mathbf{k}_{1}}^{\prime}}\right)_{j k^{\prime}} s_{\mathbf{k}_{1}}^{k^{\prime}}-2 i \epsilon_{j k i}\left(R_{\boldsymbol{\Omega}_{\mathbf{k}_{1}}}^{\chi_{\mathbf{k}_{1}}^{\prime}}\right)_{k k^{\prime}}\left(R_{\boldsymbol{\Omega}_{\mathbf{k}_{2}}^{\prime}}^{\chi_{\mathbf{k}_{2}}^{\prime}}\right)_{i i^{\prime}} s_{\mathbf{k}_{1}}^{k^{\prime}} s_{\mathbf{k}_{2}}^{i^{\prime}}\right]\right]+ \\
& +\left(E_{\mathbf{k}_{1}}^{\chi_{\mathbf{k}_{1}}}\left(E_{\mathbf{k}_{2}}^{*}\right)^{\chi_{\mathbf{k}_{2}}}\right)_{\alpha k}\left[\left(R_{\omega_{M n}(z)}^{\chi_{M n}}\right)_{k j^{\prime}} \delta_{\chi_{\mathbf{k}_{1}}^{\prime}, 0} \delta_{\chi_{\mathbf{k}_{2}}^{\prime}, 0}\left[\frac{\left\langle S^{l} S^{j^{\prime}}(z)+S^{j^{\prime}} S^{l}(z)\right\rangle}{2} \frac{n_{\mathbf{k}_{2}}-n_{\mathbf{k}_{1}}}{4}+\frac{i}{2} \epsilon_{j^{\prime} l i^{\prime \prime}}\left\langle S^{i^{\prime \prime}}(z)\right\rangle\left(\frac{n_{\mathbf{k}_{2}}+n_{\mathbf{k}_{1}}-n_{\mathbf{k}_{1}} n_{\mathbf{k}_{2}}}{4}\right)\right]+\right. \\
& +\frac{i}{2} \epsilon_{j^{\prime} l i^{\prime \prime}}\left\langle S^{i^{i^{\prime \prime}}}(z)\right\rangle\left(\delta_{j k} \delta_{k^{\prime} k^{\prime \prime}}-\delta_{j k^{\prime}} \delta_{k k^{\prime \prime}}-\delta_{j k^{\prime \prime}} \delta_{k k^{\prime}}\right)\left(R_{\boldsymbol{\omega}_{M n}(z)}^{\chi_{M n}}\right)_{j j^{\prime}}\left(R_{\boldsymbol{\Omega}_{\mathbf{k}_{1}}^{\prime}}^{\chi_{\mathbf{k}_{1}}^{\prime}}\right)_{k^{\prime} i}\left(R_{\boldsymbol{\Omega}_{\mathbf{k}_{2}}^{\prime}}^{\chi_{\mathbf{k}_{2}}^{\prime}}\right)_{k^{\prime \prime} i^{\prime}} s_{\mathbf{k}_{1}}^{i} s_{\mathbf{k}_{2}}^{i^{\prime}}+ \\
& +\frac{i}{2} \epsilon_{j k i} \frac{\left\langle S^{l} S^{j^{\prime}}(z)+S^{j^{\prime}} S^{l}(z)\right\rangle}{2}\left(R_{\boldsymbol{\omega}_{M n}(z)}^{\chi_{M n}}\right)_{j j^{\prime}}\left[\left(R_{\mathbf{\Omega}_{\mathbf{k}_{2}}^{\prime}}^{\chi_{\mathbf{k}_{2}}^{\prime}}\right)_{i l^{\prime}} \delta_{\chi_{\mathbf{k}_{1}}^{\prime}, 0} s_{\mathbf{k}_{2}}^{l^{\prime}}+\left(R_{\mathbf{\Omega}_{\mathbf{k}_{1}}^{\prime}}^{\chi_{\mathbf{k}_{1}}^{\prime}}\right)_{i l^{\prime}} \delta_{\chi_{\mathbf{k}_{2}}^{\prime}, 0} s_{\mathbf{k}_{1}}^{l^{\prime}}\right]+ \\
& \left.\left.-\frac{1}{4} \epsilon_{j k i} \epsilon_{j^{\prime} l i^{\prime \prime}}\left\langle S^{i^{\prime \prime}}(z)\right\rangle\left(R_{\boldsymbol{\omega}_{M n}(z)}^{\chi_{M n}}\right)_{j j^{\prime}}\left[\left(R_{\boldsymbol{\Omega}_{\mathbf{k}_{2}}^{\prime}}^{\chi_{\mathbf{k}_{2}}^{\prime}}\right)_{i l^{\prime}} \delta_{\chi_{\mathbf{k}_{1}}^{\prime}, 0}\left(1-n_{\mathbf{k}_{1}}\right) s_{\mathbf{k}_{2}}^{l^{\prime}}-\left(R_{\mathbf{\Omega}_{\mathbf{k}_{1}}^{\prime}}^{\chi_{\mathbf{k}_{1}}^{\prime}}\right)_{i l^{\prime}} \delta_{\chi_{\mathbf{k}_{2}}^{\prime}, 0}\left(1-n_{\mathbf{k}_{2}}\right) s_{\mathbf{k}_{1}}^{l^{\prime}}\right]\right]\right\}
\end{aligned}
$$

Finally, inserting the expression for $Q_{l \mathbf{k}_{1}}^{\kappa \mathbf{k}_{2}}$ of Eq. (34) into the quantum kinetic equations of motion (10a)-(10c) for the carrier and impurity variables, yields the desired set of ordinary differential equations for $n_{\mathbf{k}}, \mathbf{s}_{\mathbf{k}}$ and $\langle\mathbf{S}\rangle$ where the correlations are eliminated, but their effects are still accounted for.

\section{E. Numerical Implementation of the Markovian Equations of motion}

The numerical advantage of the Markov limit over the original quantum kinetic equations is mainly that, because of the $\delta$-function in Eq. (34), only those electronic states with wave vectors $\mathbf{k}_{2}$ contribute to the time evolution of electron variables with wave vector $\mathbf{k}_{1}$ that are allowed by energy conservation. Here, the total energy consists of the kinetic energy as well as Zeeman-like spindependent energies due to the impurity magnetization, the external magnetic field and the $\mathbf{k}$-dependent effective magnetic field due to the Rashba- or Dresselhaus-terms as well as the impurity Zeeman energy.

The complicated interplay of the different contributions to the total energy makes it hard to find the roots of the argument of the $\delta$-function in Eq. (34), which is necessary in order to identify the wave vectors $\mathbf{k}_{2}$ of the electronic states which are relevant for the calculation of the time evolution of electronic states with wave vector $\mathbf{k}_{1}$. In particular, the $\mathbf{k}$-dependence of the energies, the dimensionality of the $\mathbf{k}$-vector and the fact that the number of roots is in general not known turn out to be major obstacles for the direct numerical solution of Eq. (34).

Here, we solve this problem by rediscretizing the electron variables. The roots of the argument of the $\delta$ - function in Eq. (34) are given by:

$$
\bar{\omega}_{\mathbf{k}_{2}}\left(\xi_{2}\right)=\bar{\omega}_{\mathbf{k}_{1}}\left(-\xi_{1}\right)-\chi_{M n} \omega_{M n}(z)
$$

with

$$
\begin{aligned}
& \bar{\omega}_{\mathbf{k}}(\xi):=\omega_{\mathbf{k}}-\xi \Omega_{\mathbf{k}}^{\prime}, \\
& \xi \in\left\{-\frac{3}{2},-\frac{1}{2}, \frac{1}{2}, \frac{3}{2}\right\}
\end{aligned}
$$

After the space of $\bar{\omega}$ is discretized into small intervals, we create a list of discretization points in $\mathbf{k}$-space which contribute to the corresponding interval with respect to $\bar{\omega}$. Since the construction of this list has the complexity $\mathcal{O}\left(N_{k}^{2}\right)$, where $N_{k}$ is the number of discretization points of a linear dimension in the two-dimensional $\mathbf{k}$-space, and the correlations $\sum_{\mathbf{k}_{2}} Q_{l \mathbf{k}_{1}}^{\alpha \mathbf{k}_{2}}$ which enter in the equation for a single electron variable with wave-vector $\mathbf{k}_{1}$ become of the order of $\mathcal{O}\left(N_{k}^{0}\right)$ due to the $\delta$-function, the problem of solving the Markovian equations in the full $\mathbf{k}$-space is $\mathcal{O}\left(N_{k}^{2}\right)$. This provides a significant advantage over the full quantum kinetic theory which has the complexity $\mathcal{O}\left(N_{k}^{4}\right)$ for a quantum well.

\section{F. Case $N_{M n} \gg N_{e}$ without spin-orbit fields}

The Markov limit (34) of the equations of motion (10) yields quite lengthy expressions. However, these can be simplified dramatically in a case which is very common for experimentally studied DMS samples: If the number of the magnetic impurites $N_{M n}$ exceeds largely the number of quasi-free carriers $N_{e}$, such as in the case of optically excited intrinsic DMS, the impurity spin $\langle\mathbf{S}\rangle$ only changes marginally due to the influence from the 
quasi-free carriers. One can therefore assume that the impurity spin will approximately be defined by its thermal equilibrium value in the external magnetic field. In particular in the paramagnetic regime, the impurity spin will be parallel $\left(\sigma_{S}^{B}=+1\right)$ or anti-parallel $\left(\sigma_{S}^{B}=-1\right)$ to the magnetic field

$$
\langle\mathbf{S}\rangle=\sigma_{S}^{B}|\langle\mathbf{S}\rangle| \frac{\mathbf{B}}{|\mathbf{B}|}
$$

Since usually the Zeeman contribution to the energy of the magnetic ions is much stronger than the mean-field $s$ - $d$ term due to the carrier spins ${ }^{39}$, we assume that

$$
\omega_{M n}=\sigma_{M n}^{B} \omega_{M n} \frac{\mathbf{B}}{|\mathbf{B}|}
$$

and that $\boldsymbol{\omega}_{M n}$ is independent of $z$. If only electrons with small wave vectors are excited, no electric field is applied, and the sample has a rather high impurity concentration, the $s$ - $d$ interaction usually dominates over spinorbit coupling effects, so that one can neglect the latter ${ }^{21}$. Here, we shall first concentrate on this case and defer the discussion of the interplay between s-d interactions and spin-orbit coupling to section IIIB. Since the external magnetic field as well as the effective $s$ - $d$ field due the impurity spins are parallel, we find also

$$
\boldsymbol{\Omega}_{\mathbf{k}}=\omega_{e}=\sigma_{e}^{B} \omega_{e} \frac{\mathbf{B}}{|\mathbf{B}|} .
$$

Because of the $\mathbf{k}$-independence of the effective magnetic field for the carriers, the matrix $E_{\mathbf{k}_{1}} E_{\mathbf{k}_{2}}^{*}$ can be simplified to

$$
E_{\mathbf{k}_{1}} E_{\mathbf{k}_{2}}^{*}=\left(\begin{array}{cc}
1 & 0 \\
0 & R_{\boldsymbol{\omega}_{e}}
\end{array}\right)
$$

Additionally, comparing Eq. (13) with Eq. (29) yields

$$
\begin{aligned}
\left\langle S^{i^{\prime}} S^{j^{\prime}}\right\rangle= & \left\langle S^{\|^{2}}\right\rangle\left(R_{\langle\mathbf{S}\rangle}^{0}\right)_{i^{\prime} j^{\prime}}+\left\langle S^{\perp^{2}}\right\rangle\left(R_{\langle\mathbf{S}\rangle}^{+}+R_{\langle\mathbf{S}\rangle}^{-}\right)_{i^{\prime} j^{\prime}}+ \\
& +|\langle\mathbf{S}\rangle| \frac{1}{2}\left(R_{\langle\mathbf{S}\rangle}^{+}-R_{\langle\mathbf{S}\rangle}^{-}\right)_{i^{\prime} j^{\prime}}
\end{aligned}
$$

Now, the products of matrices in Eq. (34) can be evaluated using

$$
R_{\langle\mathbf{S}\rangle}^{\sigma_{S}^{B} \chi}=R_{\boldsymbol{\omega}_{M n}}^{\sigma_{M n}^{B} \chi}=R_{\boldsymbol{\omega}_{e}}^{\sigma_{e}^{B} \chi}=R_{\mathbf{B}}^{\chi}
$$

and the relation (30a). After a lengthy but straightforward calculation, we arrive at the Markov limit of the equations of motion for the occupations of the spin-up and -down subbands with respect to the direction of the external magnetic field $n_{\mathbf{k}}^{\uparrow / \downarrow}:=\frac{n_{\mathbf{k}}}{2} \pm \frac{\mathbf{B}}{|\mathbf{B}|} \cdot \mathbf{s}_{\mathbf{k}}$ and the perpendicular spin component $\mathbf{s}_{\mathbf{k}}^{\perp}:=\mathbf{s}_{\mathbf{k}}-\frac{\mathbf{B}}{|\mathbf{B}|}\left(\frac{\mathbf{B}}{|\mathbf{B}|} \cdot \mathbf{s}_{\mathbf{k}}\right)$ :

$$
\begin{aligned}
\left.\frac{\partial}{\partial t} n_{\mathbf{k}_{1} / \downarrow}\right|_{c o r} \approx & \int_{-\frac{d}{2}}^{\frac{d}{2}} d z \pi \frac{J_{s d}^{2}|\psi(z)|^{4} N_{M n} d}{\hbar^{2} V^{2}} \sum_{\mathbf{k}_{2}}\left\{\delta\left(\omega_{\mathbf{k}_{2}}-\omega_{\mathbf{k}_{1}}\right) \frac{\left\langle S^{\|^{2}}\right\rangle}{2}\left(n_{\mathbf{k}_{2} \uparrow /}-n_{\mathbf{k}_{1}}^{\uparrow / \downarrow}\right)+\delta\left(\omega_{\mathbf{k}_{2}}-\left(\omega_{\mathbf{k}_{1}} \pm\left(\sigma_{e}^{B} \omega_{e}-\sigma_{e}^{B} \omega_{M n}\right)\right)\right) \times\right. \\
& {\left.\left[\left(\left\langle S^{\perp}{ }^{2}\right\rangle \pm \sigma_{S}^{B} \frac{|\langle\mathbf{S}\rangle|}{2}\right)\left(1-n_{\mathbf{k}_{1} / \downarrow}\right) n_{\mathbf{k}_{2}}^{\downarrow / \uparrow}-\left(\left\langle S^{\perp}{ }^{2}\right\rangle \mp \sigma_{S}^{B} \frac{|\langle\mathbf{S}\rangle|}{2}\right)\left(1-n_{\mathbf{k}_{2}}^{\downarrow / \uparrow}\right) n_{\mathbf{k}_{1}}^{\uparrow / \downarrow}\right]\right\}, } \\
\left.\frac{\partial}{\partial t} \mathbf{s}_{\mathbf{k}_{1}}^{\perp}\right|_{c o r} \approx & \int_{\mathbf{k}_{2}}^{\frac{d}{2}} d z \pi \frac{J_{s d}^{2}|\psi(z)|^{4} N_{M n} d}{\hbar^{2} V^{2}} \sum\left\{-\delta\left(\omega_{\mathbf{k}_{2}}-\omega_{\mathbf{k}_{1}}\right) \frac{\left\langle S^{\|^{2}}\right\rangle}{2}\left(\mathbf{s}_{\mathbf{k}_{2}}^{\perp}+\mathbf{s}_{\mathbf{k}_{1}}^{\perp}\right)+\right. \\
& -\delta\left(\omega_{\mathbf{k}_{2}}-\left(\omega_{\mathbf{k}_{1}}+\left(\sigma_{e}^{B} \omega_{e}-\sigma_{e}^{B} \omega_{M n}\right)\right)\right)\left[\frac{1}{2}\left(\left\langle S^{\perp}{ }^{2}\right\rangle-\sigma_{S}^{B} \frac{|\langle\mathbf{S}\rangle|}{2}\right)+n_{\mathbf{k}_{2}}^{\uparrow} \sigma_{S}^{B} \frac{|\langle\mathbf{S}\rangle|}{2}\right] \mathbf{s}_{\mathbf{k}_{1}}^{\perp}+ \\
& -\delta\left(\omega_{\mathbf{k}_{2}}-\left(\omega_{\mathbf{k}_{1}}-\left(\sigma_{e}^{B} \omega_{e}-\sigma_{e}^{B} \omega_{M n}\right)\right)\right)\left[\frac{1}{2}\left(\left\langle S^{\perp}{ }^{2}\right\rangle+\sigma_{S}^{B} \frac{|\langle\mathbf{S}\rangle|}{2}\right)-n_{\mathbf{k}_{2}}^{\downarrow} \sigma_{S}^{B} \frac{|\langle\mathbf{S}\rangle|}{2}\right] \mathbf{s}_{\mathbf{k}_{1}}^{\perp}+ \\
& -\frac{1}{\pi} \frac{1}{\omega_{\mathbf{k}_{2}}-\left(\omega_{\mathbf{k}_{1}}+\left(\sigma_{e}^{B} \omega_{e}-\sigma_{e}^{B} \omega_{M n}\right)\right)}\left[\frac{1}{2}\left(\left\langle S^{\perp}\right\rangle-\sigma_{S}^{B} \frac{|\langle\mathbf{S}\rangle|}{2}\right)+n_{\mathbf{k}_{2}}^{\uparrow} \sigma_{S}^{B} \frac{|\langle\mathbf{S}\rangle|}{2}\right] \frac{\mathbf{B}}{|\mathbf{B}|} \times \mathbf{s}_{\mathbf{k}_{1}}^{\perp}+ \\
& \left.+\frac{1}{\pi} \frac{1}{\omega_{\mathbf{k}_{2}}-\left(\omega_{\mathbf{k}_{1}}-\left(\sigma_{e}^{B} \omega_{e}-\sigma_{e}^{B} \omega_{M n}\right)\right)}\left[\frac{1}{2}\left(\left\langle S^{\perp}{ }^{2}\right\rangle+\sigma_{S}^{B} \frac{|\langle\mathbf{S}\rangle|}{2}\right)-n_{\mathbf{k}_{2}}^{\downarrow} \sigma_{S}^{B} \frac{|\langle\mathbf{S}\rangle|}{2}\right] \frac{\mathbf{B}}{|\mathbf{B}|} \times \mathbf{s}_{\mathbf{k}_{1}}^{\perp}\right\},
\end{aligned}
$$

where $\left.\frac{\partial}{\partial t} n_{\mathbf{k}_{1}}^{\uparrow / \downarrow}\right|_{\text {cor }}$ and $\left.\frac{\partial}{\partial t} \mathbf{s}_{\mathbf{k}_{1}}^{\perp}\right|_{\text {cor }}$ describe the contributions to the time derivative of the respective quantities beyond the mean-field dynamics. In the case studied here, the 
total time evolution is given by:

$$
\begin{aligned}
& \frac{\partial}{\partial t} n_{\mathbf{k}_{1}}^{\uparrow / \downarrow}=\left.\frac{\partial}{\partial t} n_{\mathbf{k}_{1}}^{\uparrow / \downarrow}\right|_{c o r}, \\
& \frac{\partial}{\partial t} \mathbf{s}_{\mathbf{k}_{1}}^{\perp}=\boldsymbol{\omega}_{e} \times \mathbf{s}_{\mathbf{k}_{1}}^{\perp}+\left.\frac{\partial}{\partial t} \mathbf{s}_{\mathbf{k}_{1}}^{\perp}\right|_{c o r}, \\
& \frac{\partial}{\partial t}\langle\mathbf{S}\rangle=\boldsymbol{\omega}_{M n} \times\langle\mathbf{S}\rangle+\left.\frac{\partial}{\partial t}\langle\mathbf{S}\rangle\right|_{c o r},
\end{aligned}
$$

where $\left.\frac{\partial}{\partial t}\langle\mathbf{S}\rangle\right|_{\text {cor }}$ can be obtained by replacing $N_{M n} \int_{-\frac{d}{2}}^{\frac{d}{2}} d z$ by $-d \sum_{\mathbf{k}_{1}}$ in the r. h. s. of Eq. (40b). This follows directly from the fact that the $s$ - $d$ interaction conserves the total spin.

Note that Eqs. (40) generalize Eqs. (6) of Ref. 37 by incorporating a $\mathbf{k}$-dependent precession frequency for the electrons, an external magnetic field and the zdependence of the coupling due to the form of the envelope function of the quantum well.

Eq. (40a) can be interpreted like equations resulting from Fermi's golden rule: A spin-up electron is scattered either to another spin-up state with the same value of the kinetic energy $\hbar \omega_{\mathbf{k}}$ (term proportional to $\left.\delta\left(\omega_{\mathbf{k}_{2}}-\omega_{\mathbf{k}_{1}}\right)\right)$ or to a spin-down state with kinetic energy $\hbar \omega_{\mathbf{k}_{2}}=\hbar \omega_{\mathbf{k}_{1}}+$ $\hbar\left(\sigma_{e}^{B} \omega_{e}-\sigma_{e}^{B} \omega_{M n}\right)$ and vice-versa. To understand the latter term it is important to keep in mind that the total mean-field energy of a spin-up electron is $\hbar\left(\omega_{\mathbf{k}}+\frac{1}{2} \sigma_{e}^{B} \omega_{e}\right)$ while for a spin-down electron one finds $\hbar\left(\omega_{\mathbf{k}}-\frac{1}{2} \sigma_{e}^{B} \omega_{e}\right)$. Also, since the $s$ - $d$ interaction conserves the sum of the electron and impurity spins, a flip of an electron spin in one direction is always accompanied by a flip of an impurity spin in the opposite direction. Thus, in order to fulfill the conservation of the total mean-field energy, the energy $\hbar\left(\sigma_{e}^{B} \omega_{e}-\sigma_{e}^{B} \omega_{M n}\right)$ that is freed by an impurity mediated flip of an electron from the spin-up to the spindown state has to be compensated by a difference of the kinetic energies of the electronic states $\omega_{\mathbf{k}_{2}}-\omega_{\mathbf{k}_{1}}$.

Although Eq. (40a) for the spin-up and spin-down occupations can also be derived by Fermi's golden rule, the energy shifts in the $\delta$-functions are often not correctly accounted for in the literature ${ }^{22,32}$. The consequences are discussed in section III A. Here, the spin-flip terms of Eq. (40a) also correctly account for Pauli-blocking effects by the terms proportional to $\left(1-n_{\mathbf{k}}^{\uparrow / \downarrow}\right)$ which are usually put in by hand in a golden rule derivation. Furthermore, a golden rule treatment only allows to derive transition rates between energy eigenstates and does not provide equations governing the dynamics of the coherences between those eigenstates, i. e. the components of the electron and impurity spins perpendicular to the direction of the external magnetic field, which is given in our derivation by Eq. (40b). As in the equations for the spin-up and spin-down occupations, we find that the equations for the perpendicular spin components connect states whose difference in kinetic energies $\hbar\left(\omega_{\mathbf{k}_{2}}-\omega_{\mathbf{k}_{1}}\right)$ is either zero or $\pm \hbar\left(\sigma_{e}^{B} \omega_{e}-\sigma_{M n}^{B} \omega_{M n}\right)$. Note that in contrast to the equations for $n_{\mathbf{k}_{1}}^{\uparrow / \downarrow}$, here, we find terms pro- portional to the imaginary part of $\bar{\delta}$. While the real part leads to a rate-like damping of the perpendicular electron spin, the imaginary part yields an additional contribution to the precession frequency. Such frequency renormalizations have been extensively discussed in Ref. 28.

From Eqs. (40) one can also find decay rates for spin-up $\left(\left(\tau_{\mathbf{k}_{0}}^{\uparrow}\right)^{-1}\right)$ and spin-down $\left(\left(\tau_{\mathbf{k}_{0}}^{\downarrow}\right)^{-1}\right)$ electron states as well as the spin components parallel $\left(\left(\tau_{\mathbf{k}_{0}}^{\|}\right)^{-1}\right)$ perpendicular $\left(\left(\tau_{\mathbf{k}_{0}}^{\perp}\right)^{-1}\right)$ to the external magnetic field, if it is assumed that only very few quasi-free carriers are excited, so that one can regard only single electrons by setting $n_{\mathbf{k}_{2}}^{\uparrow / \downarrow}=$ $\delta_{\omega_{\mathbf{k}_{1}}, \omega_{\mathbf{k}_{2}}} n_{\mathbf{k}_{1}}^{\uparrow / \downarrow}$ and $\mathbf{s}_{\mathbf{k}_{2}}^{\perp}=\delta_{\omega_{\mathbf{k}_{1}}, \omega_{\mathbf{k}_{2}}} \mathbf{s}_{\mathbf{k}_{1}}^{\perp}$ :

$$
\begin{aligned}
& \left(\tau_{\mathbf{k}_{0}}^{\uparrow}\right)^{-1}=\Gamma^{-} \Theta\left(\omega_{\mathbf{k}_{0}}+\left(\sigma_{e}^{B} \omega_{e}-\sigma_{M n}^{B} \omega_{M n}\right)\right), \\
& \left(\tau_{\mathbf{k}_{0}}^{\downarrow}\right)^{-1}=\Gamma^{+} \Theta\left(\omega_{\mathbf{k}_{0}}-\left(\sigma_{e}^{B} \omega_{e}-\sigma_{M n}^{B} \omega_{M n}\right)\right), \\
& \left(\tau_{\mathbf{k}_{0}}^{\|}\right)=\left(\tau_{\mathbf{k}_{0}}^{\uparrow}\right)^{-1}+\left(\tau_{\mathbf{k}_{0}}^{\downarrow}\right)^{-1} \\
& \left(\tau_{\mathbf{k}_{0}}^{\perp}\right)^{-1}=\Gamma^{0}+\frac{1}{2}\left[\left(\tau_{\mathbf{k}_{0}}^{\uparrow}\right)^{-1}+\left(\tau_{\mathbf{k}_{0}}^{\downarrow}\right)^{-1}\right],
\end{aligned}
$$

with

$$
\begin{aligned}
& \Gamma^{0}=I \pi D^{2 D} \frac{J_{s d}^{2} N_{M n}}{\hbar^{2} V^{2}}\left\langle S^{\|^{2}}\right\rangle, \\
& \Gamma^{ \pm}=I \pi D^{2 D} \frac{J_{s d}^{2} N_{M n}}{\hbar^{2} V^{2}}\left(\left\langle S^{\perp^{2}}\right\rangle \pm \sigma_{S}^{B} \frac{|\langle\mathbf{S}\rangle|}{2}\right), \\
& I=d \int_{-\frac{d}{2}}^{\frac{d}{2}} d z|\psi(z)|^{4},
\end{aligned}
$$

where $\Theta(x)$ is the Heaviside step function.

Thus, the main effect of the frequency shifts due to the precession of the correlations is the opening and closing of decay channels due to the corresponding step functions which originate from the step of the two-dimensional density of states at $\omega_{\mathbf{k}=0}$.

\section{RESULTS}

\section{A. Magnetic Field Dependence of the Spin Transfer Rates}

Now, we compare the theory derived in the present paper with the different treatments of the $s$ - $d$ interaction presented by other groups. To this end, we focus on the case without spin-orbit interactions and $N_{M n} \gg N_{e}$, so that the correlation induced changes in the carrier variables can be described by Eqs. (40). Often in the literature rates for the carrier-impurity spin transfer dynamics are obtained from Fermi's golden rule ${ }^{25,29-31}$. In two-dimensional systems one finds in absence of magnetic 
fields:

$$
\begin{aligned}
\frac{\partial}{\partial t} s_{\omega_{1}}^{i}= & -I \pi \frac{J_{s d}^{2}}{\hbar^{2}} \frac{N_{M n}}{V^{2}} \frac{2}{3}(S(S+1)) \times \\
& \int d \omega D^{2 D}(\omega) \delta\left(\omega-\omega_{1}\right) s_{\omega}^{i}=-\tau_{F G R} s_{\omega_{1}}^{i} \\
\tau_{F G R}= & I \pi \frac{J_{s d}^{2}}{\hbar^{2}} \frac{N_{M n}}{V^{2}} \frac{2}{3}(S(S+1)) \frac{A m^{*}}{2 \pi \hbar}
\end{aligned}
$$

where we assume isotropy so that the carrier spin variables are independent of the angle of the wave vector and can equivalently be described by $s_{|\mathbf{k}|}^{i}$ or $s_{\omega}^{i}$, with the kinetic energy $\hbar \omega=\frac{\hbar^{2}|\mathbf{k}|^{2}}{2 m}$. $\tau_{F G R}$ is Fermi's golden rule spin-transfer rate at $B=0$. In contrast, if an external magnetic field is applied, the conduction band is energetically split by $\sigma_{e}^{B} \omega_{e}$. This leads to the appearance of an additional energy offset in the $\delta$-function. In our treatment, we also find an energy offset corresponding to the impurity Zeeman splitting $\sigma_{M n}^{B} \omega_{M n}$ which is necessary for the simultaneous conservation of the total carrier and impurity energy as well as the total spin. Furthermore, Fermi's golden rule is only able to predict transitions between energy eigenstates, whereas it makes no statement about the transfer of the carrier spin components perpendicular to the quantization axis. The distinction between parallel and perpendicular components does not arise for $B=0$, since in this case all directions are equivalent. Additionally, the factor $S(S+1)$ has to be modified in the presence of a magnetic field that causes a non-zero paramagnetic impurity magnetization.

In particular, the energetic offset caused by the impurity Zeeman splitting is often overlooked in studies based on the golden rule approach ${ }^{22,29}$. In Ref. 22, which is based on the kinetic spin Bloch equations (KSBEs), even the band splitting $\sigma_{e}^{B} \omega_{e}$ is disregarded, but the magnetic field dependence of the second moments of the impurity spin, which enters in the rates, was kept. There are also studies $^{25,26,30}$ that explicitly include the band splitting as well as the impurity Zeeman terms, but since there the rates are derived by Fermi's golden rule, no expression for the perpendicular spin transfer component was given.

In this context, one particularly notable theoretical derivation of magnetic field dependent carrier-impurity spin transfer rates was given by Semenov in Ref. 32, which is based on a projection operator method. There, the electron spins are treated as a subsystem which interacts with a bath of impurity ions. In Ref. 32, it was assumed that the electron density matrix can be factorized into one part accounting for the spin degree of freedom and the $\mathbf{k}$-dependent part, which is described by a Fermi distribution. Tracing out the $\mathbf{k}$-dependent part of the carrier density matrix as well as the impurity system, rates were obtained for the spin degree of freedom of the carriers. In contrast to the theory of the present article, where only energetic shifts associated with the spin flip-flop processes of the form $\left|\sigma_{e}^{B} \omega_{e}-\sigma_{M n}^{B} \omega_{M n}\right|$ appear, the projection operator method of Ref. 32 also finds

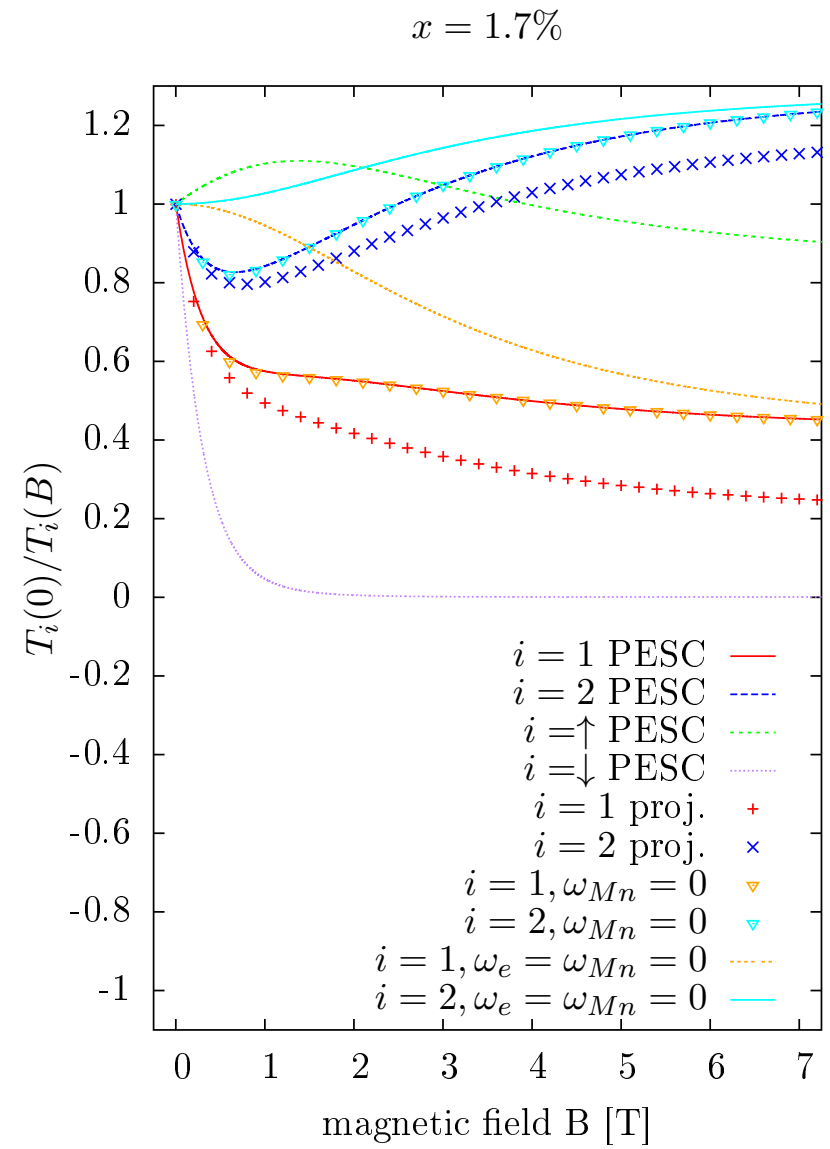

FIG. 1. Magnetic field dependence of the parallel $(i=1)$ and perpendicular $(i=2)$ spin transfer rates normalized with respect to $B=0$ in a $8 \mathrm{~nm}$ wide $\mathrm{Cd}_{0.983} \mathrm{Mn}_{0.017}$ Te quantum well at temperature $T=4 \mathrm{~K}$. Red and blue lines (PESC) represent rates according to the theory of the present article [Eqs. (45)] and red and blue crosses show the rates calculated by the projection operator method (proj.) of Ref. 32 . Furthermore, cyan and orange triangles and lines show the results of Eqs. (45), when the energetic shifts due to the Zeeman impurity splittings in spin flip-flop processes $\left(\omega_{M n}=0\right)$ or additionally the spin-splittings $\left(\omega_{e}=\omega_{M n}=0\right)$ are neglected. $T_{\uparrow}$ and $T_{\downarrow}$ are the relaxation rates of spin-up and spin-down occupations, respectively.

terms proportional to $\left|\sigma_{e}^{B} \omega_{e}+\sigma_{M n}^{B} \omega_{M n}\right|$. As mentioned earlier, such energy shifts are in conflict with the conservation of the total carrier and impurity energy. We trace the appearance of the energy non-conserving terms in Ref. 32 back to the fact that, there, only the positive frequency component of the electron spin precession was regarded, whereas the negative frequency component explicitly shows up in the theory of the present article and leads to a cancellation of terms in the expression for the correlations which oscillate with $\pm\left(\sigma_{e}^{B} \omega_{e}+\sigma_{M n}^{B} \omega_{M n}\right)$.

Having discussed the different expressions for the magnetic field dependence of the carrier-impurity spin transfer rates that can be found in the literature, we compare them at the example of the situation discussed in Ref. 32 . There, it was assumed that the spectral electron distri- 


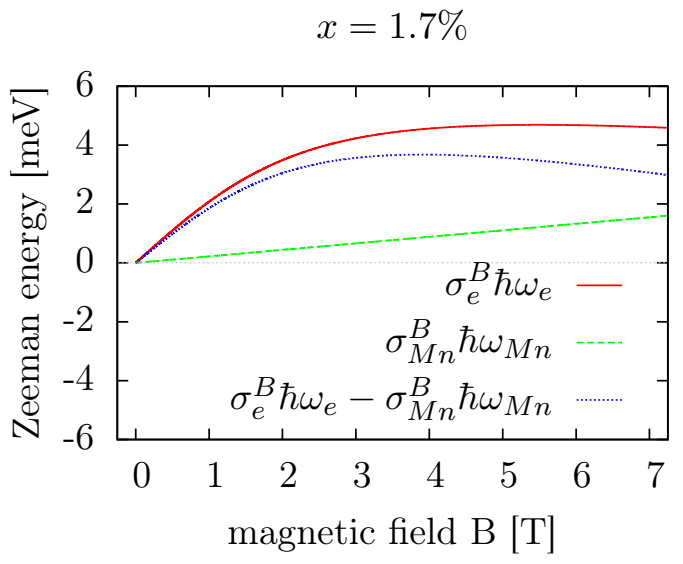

FIG. 2. Magnetic field dependence of the electron (red solid line) and impurity Zeeman energy (green dashed line) as well as their difference (blue dotted line) for a DMS quantum well (same parameters as for Fig. 1).

bution is

$$
n^{\uparrow}(\omega)=n^{\downarrow}(\omega) \propto e^{-\frac{\omega}{T}}
$$

for some carrier temperature $T$, irrespective of the spinsplit subband. With this assumption, the decay rate of the total parallel $\left(T_{1}^{-1}\right)$ and perpendicular $\left(T_{2}^{-1}\right)$ carrier spin with respect to the magnetic field direction can be obtained from Eqs. (42) of the present theory:

$$
\begin{aligned}
& T_{1}^{-1} \propto \int_{0}^{\infty} d \omega e^{-\frac{\omega}{T}}\left(\tau^{\|}(\omega)\right)^{-1} \propto T_{\uparrow}^{-1}+T_{\downarrow}^{-1} \\
& T_{2}^{-1} \propto \int_{0}^{\infty} d \omega e^{-\frac{\omega}{T}}\left(\tau^{\perp}(\omega)\right)^{-1} \propto \Gamma^{0}+\frac{1}{2}\left(T_{\uparrow}^{-1}+T_{\downarrow}^{-1}\right)
\end{aligned}
$$

$T_{\uparrow}^{-1} \propto \int_{0}^{\infty} d \omega e^{-\frac{\omega}{T}}\left(\tau^{\uparrow}(\omega)\right)^{-1} \propto \Gamma^{-} \min \left(1, e^{\frac{\sigma_{e}^{B} \omega_{e}-\sigma_{M n}^{B} \omega_{M n}}{T}}\right)$

$T_{\downarrow}^{-1} \propto \int_{0}^{\infty} d \omega e^{-\frac{\omega}{T}}\left(\tau^{\downarrow}(\omega)\right)^{-1} \propto \Gamma^{+} \min \left(1, e^{-\frac{\sigma_{e}^{B} \omega_{e}-\sigma_{M n}^{B} \omega_{M n}}{T}}\right)$

where also the values for the decay rate of the spin-up $\left(T_{\uparrow}^{-1}\right)$ and spin-down occupations $\left(T_{\downarrow}^{-1}\right)$ are given explicitly. For $B=0$, the rates $T_{1}^{-1}=T_{2}^{-1}=2 T_{\uparrow}^{-1}=2 T_{\downarrow}^{-1}$ coincide with the rate calculated by Fermi's golden rule $\tau_{F G R}$, which defines the normalization of the rates in Eq. (45).

Fig. 1 shows the magnetic field dependence of the parallel and perpendicular spin transfer rates according to Eqs. (45) with the parameters of Ref. 32, where a $d=8$ nm wide $\mathrm{Cd}_{0.983} \mathrm{Mn}_{0.017} \mathrm{Te}$ quantum well was considered at $T=4 \mathrm{~K}$. The value for the coupling constant is
$J_{s d}=15 \mathrm{meVnm}^{3}$ and the electron and Mn g-factors are $g_{e}=-1.77$ and $g_{M n}=2.0$ respectively. The present theory predicts that the parallel spin transfer rate $T_{1}^{-1}$ first decays fast from $B=0$ to $B \approx 1 \mathrm{~T}$, then levels off. The perpendicular spin transfer rate $T_{2}^{-1}$ first decays with increasing magnetic field, reaches a minimum at $B \approx 1 \mathrm{~T}$ and finally increases again. This behaviour of $T_{1}^{-1}$ and $T_{2}^{-1}$ can be explained by considering the rates $T_{\uparrow}^{-1}$ and $T_{\downarrow}^{-1}$ separately, together with the values of the energy shifts $\sigma_{e}^{B} \omega_{e}-\sigma_{M n}^{B} \omega_{M n}$ presented in Fig. 2. The meanfield impurity energy $\hbar \sigma_{e}^{B} \omega_{M n}$ is mainly dominated by its Zeeman energy and therefore increases linearly with $B$. In contrast, the mean-field carrier energy $\hbar \sigma_{e}^{B} \omega_{e}$ is strongly modified by a contribution proportional to a $S=\frac{5}{2}$ Brillouin-function due to the impurity magnetization, which starts linearly in $B$ but begins to saturate at $B \approx 2 \mathrm{~T}$. For high magnetic fields $(B>6 \mathrm{~T})$, $\hbar \sigma_{e}^{B} \omega_{e}$ decreases again, when the impurity magnetization is fully saturated and the negative electron g-factor becomes important. Although $\sigma_{e}^{B} \omega_{e}-\sigma_{M n}^{B} \omega_{M n}$ eventually becomes negative for very high magnetic fields (not shown in Fig. 2), for typical experimentally accessible fields, it is mostly positive and increases linearly up to $B \approx 2 \mathrm{~T}$, just like $\sigma_{e}^{B} \omega_{e}$.

It follows from Eq. (45c) that $T_{\downarrow}^{-1}$ decreases approximately exponentially with $B$ in the regime where $\sigma_{e}^{B} \omega_{e}-\sigma_{M n}^{B} \omega_{M n}$ increases linearly. Therefore, we find that the spin-splitting introduced by the external magnetic field closes the transfer channel $T_{\downarrow}^{-1}$. In the case studied here, the magnetic field dependence of the rate $T_{\uparrow}^{-1}$ comes exclusively from the prefactor, since due to the positive value of $\sigma_{e}^{B} \omega_{e}-\sigma_{M n}^{B} \omega_{M n}$ the corresponding transfer channel is maximally open. Noting that

$$
\begin{aligned}
\Gamma^{0}(B \rightarrow \infty) & \rightarrow \frac{15}{14} \tau_{F G R}, \\
\Gamma^{+}(B \rightarrow \infty) & \rightarrow 0, \\
\Gamma^{-}(B \rightarrow \infty) & \rightarrow \frac{3}{7} \tau_{F G R},
\end{aligned}
$$

we find that $T_{1}^{-1}$ approaches $\frac{5}{8} \tau_{F G R}$ and $T_{2}^{-1} \rightarrow$ $\frac{9}{7} \tau_{F G R} \approx 1.29 \tau_{F G R}$ for large values of $B$.

The magnetic field dependence of rates predicted from the projection operator method of Ref. 32 is qualitatively similar to that of the present theory, as can be seen in Fig. 1. However, they suggest quantitatively smaller values for the rates, with deviations of the order of $\sim 0.2 \tau_{F G R}$. In the case studied here, the offset due to the impurity Zeeman splitting $\sigma_{M n}^{B} \omega_{M n}$ plays a less significant role, so that the rates calculated neglecting these terms (triangles in Fig. 1) coincide with the caculation which conserves the total energy. However, neglecting the spin-splittings $\sigma_{M n}^{B} \omega_{M n}$ is found to lead to the correct rates only for large values of the magnetic field while for smaller magnetic fields qualitative features, such as the minimum in $T_{2}^{-1}$, are not obtained.

In our analysis of the magnetic field dependence of the spin transfer rates it was important that $\sigma_{e}^{B} \omega_{e}-$ 


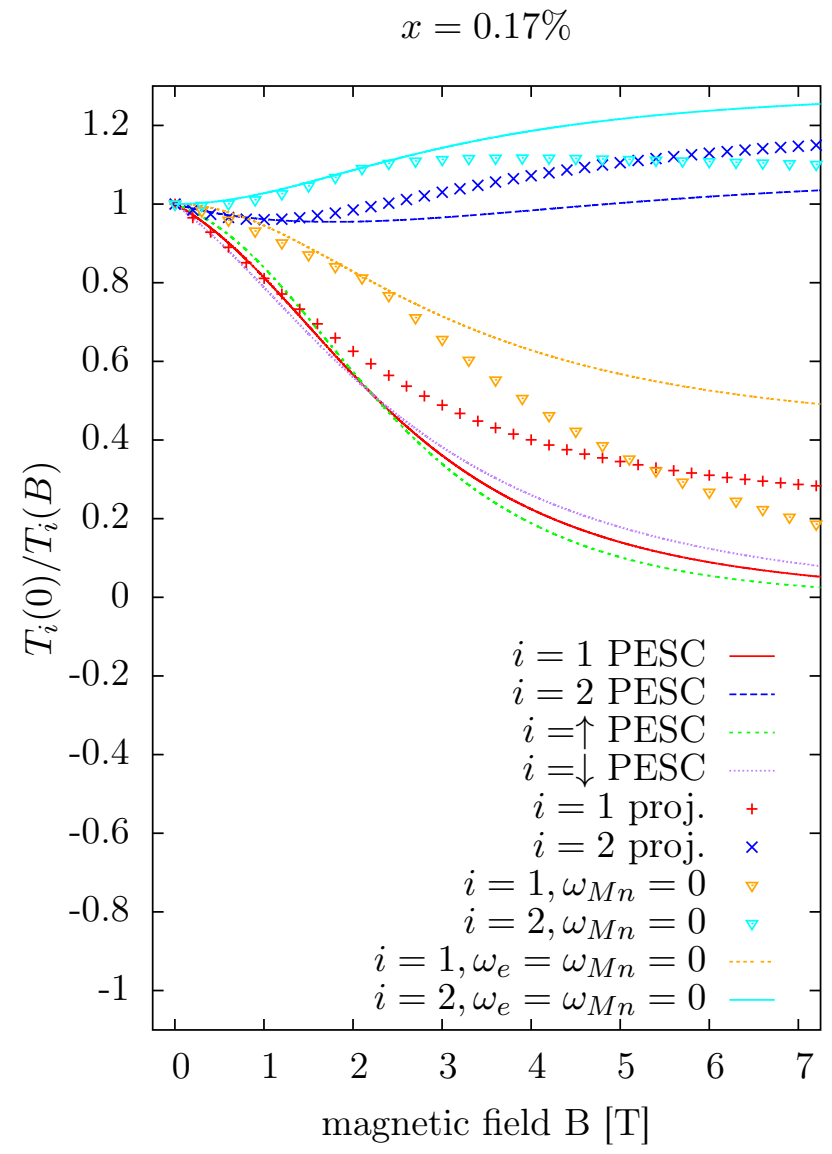

FIG. 3. Magnetic field dependence of spin transfer rates for a $\mathrm{Cd}_{0.9983} \mathrm{Mn}_{0.0017}$ Te quantum well [cf. Fig. 1]

$\sigma_{M n}^{B} \omega_{M n}>0$. The situation can change significantly, if this is not the case. In order to study this regime of parameters, we repeat the same calculations shown in Figs. 1 and 2 but we assume a Mn concentration $x=0.17 \%$ which is smaller by a factor of 10 than in the previous calculations. The results are displayed in Figs. 3 and 4 respectively. We find in Fig. 4 that now also the electron spin-splitting is dominated by the Zeeman term and the mean-field contribution from the impurity magnetization is rather small. In particular, one finds that $\sigma_{e}^{B} \omega_{e}-\sigma_{M n}^{B} \omega_{M n}$ is now negative for all values of $B>0$. This fact has immediate consequences on the magnetic field dependence of the spin transfer rates. The main qualitative difference between the rates shown in Fig. 3 and in the previous case is that now the parallel spin transfer rate $T_{1}^{-1}$ decays to zero for large values of $B$. Here, the spin transfer channel corresponding to $T_{\uparrow}^{-1}$ is closed due to the energy splitting, whereas $T_{\downarrow}^{-1}$ decreases to zero, because the prefactor $\Gamma^{+}$tends to zero for $B \rightarrow \infty$. The physical reason for this behaviour is that due to the negativity of $\sigma_{e}^{B} \omega_{e}-\sigma_{M n}^{B} \omega_{M n}$ spin-flips from the spin-up to the spin-down band face an energy penalty, while a flip from the spin-down to the spin-up band would require a corresponding decrease

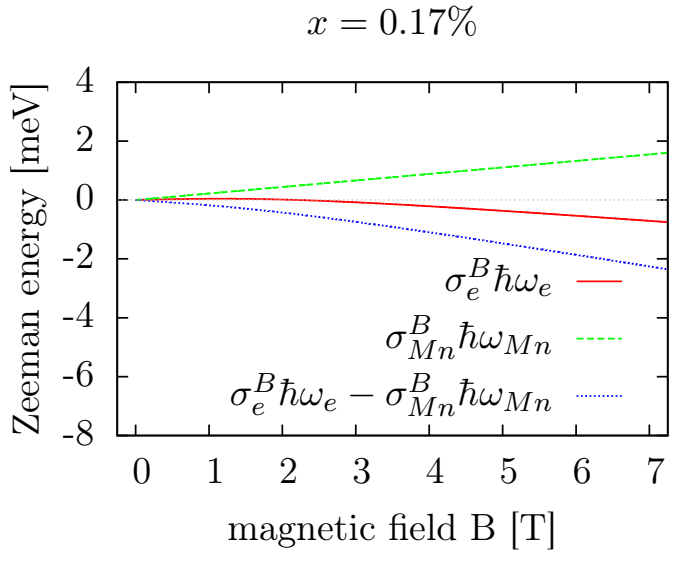

FIG. 4. Magnetic field dependence of the Zeeman energies for a $\mathrm{Cd}_{0.9983} \mathrm{Mn}_{0.0017}$ Te quantum well [cf. Fig. 2]

of an impurity spin in order to satisfy the spin conservation. However, for $B \rightarrow \infty$ the impurity spins are already fully aligned antiparallel to the magnetic field, so that this spin-flip is also forbidden. The magnetic field dependence of the perpendicular spin transfer rate $T_{2}^{-1}$ for $x=0.17 \%$ is quantitatively similar to the case of $x=1.7 \%$. However, here, the asymptotic value for strong magnetic fields is $T_{2}^{-1}(B \rightarrow \infty) \rightarrow \frac{15}{14} \tau_{F G R}$.

For the smaller impurity concentrations, the projection operator method of Ref. 32 overestimates the spin transfer rates. Fig. 3 also shows that, in this case, neglecting the impurity Zeeman terms leads to significant deviations from the energy-conserving rates.

In order to establish a connection between the theories discussed above and the experimentally determined electron spin relaxation rates, it has to be noted that in most magneto-optical experiments on II-VI DMS quantum wells so far (cf. Ref. 3 and references therein) the pump laser is tuned to the electron-heavy-hole exciton energy. To model these experiments also the Coulomb correlations between electrons and holes have to be taken into account, which is beyond the scope of the present article. It was found in Ref. 3 that different groups consistently measured perpendicular electron spin relaxation rates $T_{2}^{-1}$ which are about 5 times larger than $\tau_{F G R}$ at $B=0$. This discrepancy can be understood by the fact that the effective electron mass has to be replaced by the exciton mass in the expression for the rate $\tau_{F G R}^{-1}{ }^{48}$, which yields an increase of the rate by a factor of $\sim 4.6$ in the case of CdMnTe. Nevertheless, the finding of the present article that the rate $T_{2}^{-1}$ varies only weakly with the magnetic field and stays essentially within $30 \%$ of $\tau_{F G R}^{-1}$ is consistent with the tendency of most of the experimental results summarized in Ref. 3. However, especially for samples with low impurity concentration at low temperatures, there are also some experiments which measured a maximum (instead of a minimum predicted by the present theory) of the magnetic field dependence of the perpendicular spin transfer rate as well as changes in the rate which span about one order of mag- 
nitude of its value at $B=0$, which was suggested $^{3}$ to stem from local fluctuations of the impurity magnetization. In order to distinguish these imhomogeneity effects from Coulomb correlation effects we suggest experiments where the pump pulse is tuned to energies well above the exciton resonance.

\section{B. Interplay between $s-d$ and Rashba Interactions}

The fact that in the derivation of Eq. (34) the $\mathbf{k}$ dependence of an effective magnetic field was taken into account makes it possible to discuss the interplay between the spin-orbit coupling and the $s$ - $d$ interaction on a rigorous microscopic basis, where the spin-orbit interaction also acts during $s$ - $d$ scattering events. In earlier works, the interplay between these effects was studied $^{21,49}$, where only the direct effects of the spin-orbit coupling on the electron spins was considered, yielding an additional $\mathbf{k}$-dependent contribution to the mean-field precession frequency, whereas the dynamics of the correlations was not modified, i. e., the spin-orbit interaction was only accounted for between $s$ - $d$ scattering events. It was found that already on a mean-field level, the carrier spin dephasing due to the $\mathbf{k}$-dependence of the precession frequencies can be strongly suppressed by a motionalnarrowing-type mechanism caused by the precession in the mean field of the impurity magnetization. Furthermore, it was argued that both mechanisms can be tuned in a wide range, especially in $\mathrm{Hg}_{1-x-y} \mathrm{Cd}_{y} \mathrm{Mn}_{x}$ Te quantum wells with applied electric fields. In this material, the strength of the $s$ - $d$ interaction is determined by the Mn concentration $x$, while the $\mathrm{Cd}$ concentration $y$ can be used to change the gap between conduction and valence bands which controls the strength of the Rashba ${ }^{19}$ field. When both types of interaction are similarly important, a complex oscillatory time evolution of the carrier spin was found, which is absent when either one of the interactions dominates.

Now, the question arises whether neglecting the effects of the Rashba field on the dynamics of the correlations is indeed a good approximation or if qualitative changes have to be expected if they are accounted for. We study this question in a case in which the strengths of the Rashba and the $s$ - $d$ interactions are comparable. We consider a $d=20 \mathrm{~nm}$ wide $\mathrm{Hg}_{1-x-y} \mathrm{Cd}_{y} \mathrm{Mn}_{x}$ Te quantum well with electric and magnetic fields applied along the growth direction $z$. The voltage drop between the barriers of the quantum well leads to a strong Rashba interaction of the form

$$
H_{R}=2 \hbar \alpha_{R} \sum_{\mathbf{k} \sigma \sigma^{\prime}}\left(k_{y} s_{\sigma \sigma^{\prime}}^{x}-k_{x} s_{\sigma \sigma^{\prime}}^{y}\right) c_{\mathbf{k} \sigma}^{\dagger} c_{\mathbf{k} \sigma^{\prime}},
$$

where we assume a value of $\alpha_{R}=4.87 \mathrm{meVnm}^{21}$.

Further parameters that enter the calculation are the effective mass $m^{*}=0.093 m_{0}$, the $s$ - $d$ coupling constant $J_{s d}=15 \mathrm{meV} \mathrm{nm}^{3}$, the lattice constant $a=0.645 \mathrm{~nm}$ and the Mn concentration $x=7 \%$. The initial Mn state

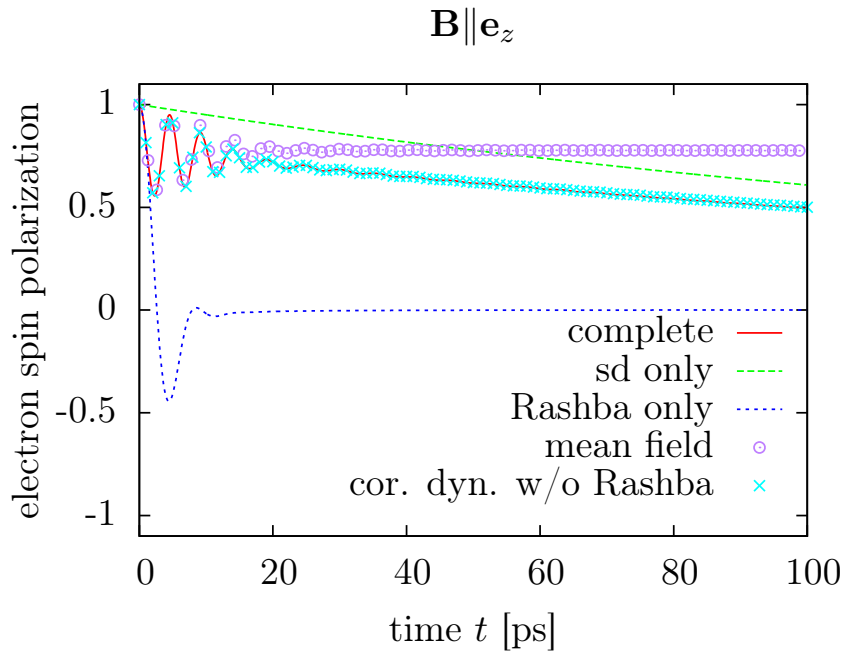

FIG. 5. Time evolution of the total electron spin polarization after spin polarized optical excitation in a magnetic field perpendicular to the quantum well plane (cf. text for parameters). The red solid line describes the results according to Eqs. (10b) and (10c) with the Markovian expression for the correlations from Eq. (34). The green dashed line corresponds to a calculation without Rashba coupling, where only the $s$-d interaction is considered. The blue dotted line presents the results of the case in which only the Rashba interaction is present. The mean-field calculation, which is obtained by dropping the correlations completely, is shown as the purple circles. The cyan crosses describe the results where the effects due to the Rashba interaction on the dynamics of the carrierimpurity correlations are neglected, so that in addition to the mean-field terms, the time derivative of the carrier variables obtains the correlation induced contribution of Eqs. (40).

is modelled by a thermal equilibrium state following a Brillouin function with temperature $T=4 \mathrm{~K}$ in an external magnetic field pointing in the $-z$-direction with $|\mathbf{B}|=50 \mathrm{mT}$. The g-factors for impurities and conduction band electrons are $g_{M n}=2$. and $g_{e}=-1.5$, respectively. Furthermore, as we consider an intrinsic DMS where the quasi-free carriers originate purely from optical excitation, $N_{M n} \gg N_{e}$ is clearly fulfilled, so that we can neglect the back-action of the carriers on the impurities. Thus, the Mn magnetization remains homogeneous, which allows us to integrate along the growth direction yielding a factor of $I=1.5$. The initial electron spin was modelled by a Gaussian distribution in the spin-up band centered at the band edge with standard deviation $E_{s}=1 \mathrm{meV}$ corresponding to a $\sigma^{-}$polarized laser with pulse duration $(\mathrm{FWHM}) \sim 140$ fs. For these parameters, the mean-field energy splitting caused by the impurity magnetization is $\sim-0.75 \mathrm{meV}$ (the spin-upsubband is energetically favored), while the strength of the Rashba interaction for an electron with kinetic energy $\frac{\hbar^{2} k_{0}^{2}}{2 m^{*}}=1 \mathrm{meV}$ is $2 \hbar \alpha_{R} k_{0} \sim 0.89 \mathrm{meV}$. Here, the Zeeman terms yield significantly smaller contributions of $g_{e} \mu_{B}|\mathbf{B}| \approx-0.004 \mathrm{meV}$ and $g_{M n} \mu_{B}|\mathbf{B}| \approx 0.006 \mathrm{meV}$ to the respective spin splittings. 


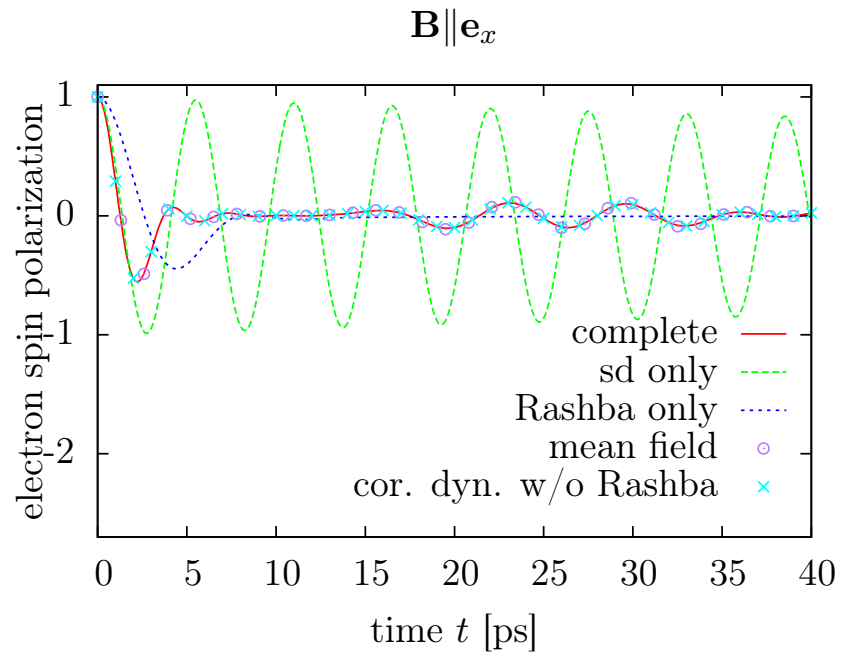

FIG. 6. Time evolution of the total electron spin polarization after spin polarized optical excitation in a magnetic field parallel to the quantum-well plane (cf. Fig. 5)

Fig. 5 shows the results of numerical simulations for this set of parameters. As reported earlier ${ }^{21}$, the Rashba interaction alone (blue dashed line) leads to a fast dephasing of the carrier spins. If additionally magnetic impurities with a finite magnetization are present, already a mean-field treatment (purple circles) can lead to a strong suppression of the dephasing by motional narrowing caused by the precession of the carrier spin in the mean field of the impurity magnetization. Without the Rashba interaction, the $s$ - $d$ interaction causes a spin transfer from the carriers to the impurities which can be seen in Fig. 5 as an exponential decay to a non-vanishing equilibrium value. In the previous studies ${ }^{21}$, the correlation induced spin transfer was combined with the meanfield precession, but the effects of the Rashba interaction on the dynamics of the correlations were neglected (here shown as cyan crosses). In Fig. 5, also the complete carrier spin dynamics is shown, where the Rashba interaction is explicitly accounted for in the calculation of the correlations (red solid line). By comparing both calculations, it can be seen that the total carrier spin is hardly influenced by the effects of the Rashba spin-orbit coupling on the correlation dynamics. The same result is also obtained for the situation where the magnetic field is applied parallel to the quantum well plane, as shown in Fig. 6.

Similar to the fact that the precession-type motion of the correlations discussed so far leads to changes in the kinetic energy of scattered carriers, also the Rashba interaction enforces a precession of the correlations resulting in corresponding changes in the electron energies. In Fig. 7 the carrier occupations at $t=0$ and $t=50 \mathrm{ps}$ are shown for calculations with and without accounting for the Rashba effect on the correlation dynamics for the situation described in Fig. 5 with magnetic field parallel to the growth direction. Without

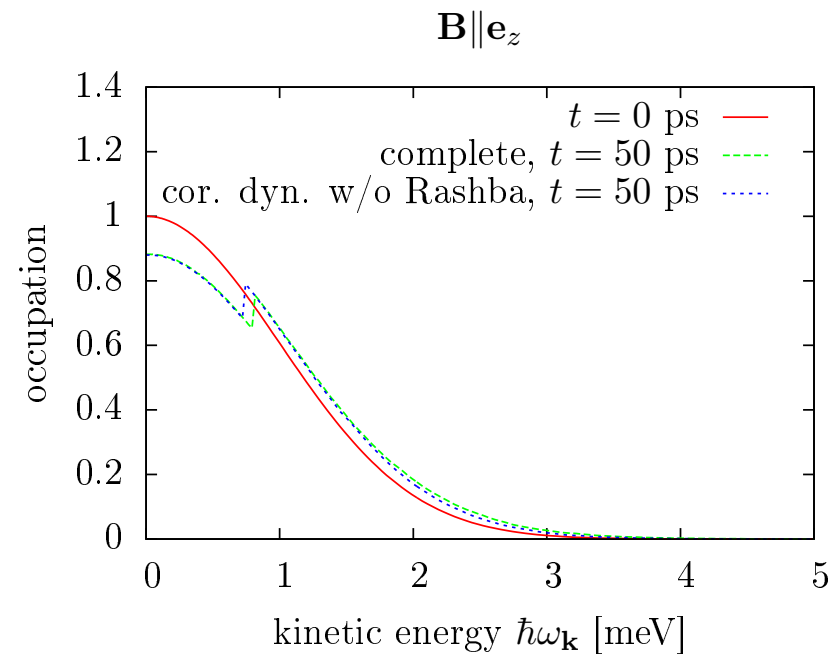

FIG. 7. Kinetic energy dependence of the occupation of carrier states at times $t=0 \mathrm{ps}$ and $t=50 \mathrm{ps}$ for the calculations shown in Fig. 5.

the Rashba interaction, the kinetic energy dependence of the occupations at $t=50 \mathrm{ps}$ shows a distinctive step at $\hbar \omega_{\mathbf{k}}=\left|\hbar \sigma_{e}^{B} \omega_{e}-\hbar \sigma_{M n}^{B} \omega_{M n}\right|$ which corresponds to a redistribution of carriers with an excess energy in the spin degree of freedom to states with higher kinetic energies. When the Rashba coupling is turned on, the step shifts towards slightly higher kinetic energies. This can be explained by the fact that in the configuration with a magnetic field along the growth direction and a Rashba field in the quantum well plane the energy eigenvalues of an electron with wave vector $\mathbf{k}$ are

$$
E_{ \pm}=\hbar \omega_{\mathbf{k}} \pm \frac{1}{2} \hbar \sqrt{\left(2 \alpha_{R}|\mathbf{k}|\right)^{2}+\left(\sigma_{e}^{B} \omega_{e}\right)^{2}} .
$$

Including the shifts due to the impurity Zeeman splittings, the step in the kinetic energy dependence of the occupation is therefore shifted to $\hbar \sqrt{\left(2 \alpha_{R}|\mathbf{k}|\right)^{2}+\left(\sigma_{e}^{B} \omega_{e}-\sigma_{M n}^{B} \omega_{M n}\right)^{2}}$. However, the shift of the energy splitting is too small to cause a significant impact on the time evolution of the total spin.

\section{Connection to the theory of collective carrier-impurity precession modes in DMS}

In the derivation of the theory, the $z$-dependence of the carrier envelope function was taken into account. We see from Eqs. (42) that one effect of this $z$-dependence is that the spin transfer rate obtains the prefactor $I$. Assuming a constant linear impurity density $\frac{N_{M n}}{d}$, a constant $z$ envelope yields a value of $I=1$ while the extreme case of a quantum well with infinite barriers yields $I=\frac{3}{2}$. This effect has also been found in previous studies of $\operatorname{DMS}^{3,15,32}$.

Like the spin transfer rates, also the electron spin precession is influenced by the $z$-dependence of the envelope 
of the electron wave function. In particular, it can be seen from Eq. (11c) that the contribution to the electron spin precession frequency from the impurity spin is proportional to $\int_{-\frac{d}{2}}^{\frac{d}{2}} d z|\psi(z)|^{2}\langle\mathbf{S}(z)\rangle$. Thus, the impurity spin as a function of $z$ can be decomposed into this mode which couples to the electron spin precession and $N_{M n}-1$ orthogonal modes, which do not influence the electron spins directly on a mean-field level. In the parameter regime where the precession frequencies of the electron and impurity spins almost coincide, the coupling between the above impurity mode and the electron spin is particularly large, leading to an avoided crossing indicating a collective motion of impurity and carrier spins. This fact has been discussed in a number of recent articles by different groups ${ }^{6,11,39,50-52}$. In these works, however, the carrier-impurity correlations have been disregarded.

In the following, we will derive equations describing the situation studied, e.g., in Ref. 39 taking the effects due to the correlations into account. There, a n-type CdMnTe quantum well in an external magnetic field parallel to the quantum well plane ( $x$-direction) was considered, leading to equilibrium values of the impurity and carrier spins antiparallel to the magnetic field. A circularly polarized pump beam induces electron-hole pairs with spin polarization along the $z$-direction. During the decay of the hole spins on a timescale of $\sim 5 \mathrm{ps}$, the impurity magnetization precesses around the $p$ - $d$ exchange field of the holes, causing a small tilt of the impurity spins away from the equilibrium $x$-axis into the $y$-axis. The optically induced electron spins contribute to the $z$-component of the total carrier spin. Thus, after the holes are decayed, one ends up with a situation where the impurity and carrier spins precess around each other.

The fact that the spin components perpendicular to the equilibrium values are small compared with the parallel components allows one to linearize Eqs. (40) and (41) with the expression for the rates from Eq. (42):

$$
\begin{aligned}
\frac{\partial}{\partial t} \mathbf{s}_{>/<}^{\perp} & =\frac{g_{e} \mu_{B}}{\hbar} \mathbf{B} \times \mathbf{s}_{>/<}^{\perp}+\frac{J_{s d} N_{M n}}{\hbar V} \mathbf{S}^{x,(1)} \times \mathbf{s}_{>/<}^{\perp}-\frac{J_{s d} N_{M n}}{\hbar V} \mathbf{s}_{>/<}^{x} \times \mathbf{S}^{\perp,(1)}-\frac{1}{d} \int_{-\frac{d}{2}}^{\frac{d}{2}} d z \Gamma^{>/<}(z) \mathbf{s}_{>/<}^{\perp} \\
\frac{\partial}{\partial t} \mathbf{S}^{\perp,(j)} & =\frac{g_{M n} \mu_{B}}{\hbar} \mathbf{B} \times \mathbf{S}^{\perp,(j)}-\frac{J_{s d}}{V \hbar} \mathbf{S}^{x,(j+1)} \times\left(\mathbf{s}_{>}^{\perp}+\mathbf{s}_{<}^{\perp}\right)+\frac{J_{s d}}{V \hbar}\left(\mathbf{s}_{>}^{x}+\mathbf{s}_{<}^{x}\right) \times \mathbf{S}^{\perp,(j+1)}+ \\
& +d^{j-1} \int_{-\frac{d}{2}}^{\frac{d}{2}} d z|\psi(z)|^{2 j}\left(\Gamma^{>}(z) \mathbf{s}_{>}^{\perp}+\Gamma^{<}(z) \mathbf{s}_{<}^{\perp}\right) \\
\Gamma(\mathbf{k}, z): & =\pi \frac{A m^{*}}{2 \pi \hbar} \frac{J_{s d}^{2} N_{M n}}{\hbar^{2} V^{2}} d^{2}|\psi(z)|^{4}\left[\left\langle S^{\|^{2}}\right\rangle+\left(\frac{\left\langle S^{\perp}\right\rangle}{2}-\sigma_{S}^{B} \frac{|\langle\mathbf{S}\rangle|}{4}\right) \Theta\left(\omega_{\mathbf{k}}+\left(\sigma_{e}^{B} \omega_{e}-\sigma_{e}^{B} \omega_{M n}(z)\right)\right)+\right. \\
& \left.+\left(\frac{\left\langle S^{\perp}\right\rangle}{2}+\sigma_{S}^{B} \frac{|\langle\mathbf{S}\rangle|}{4}\right) \Theta\left(\omega_{\mathbf{k}}-\left(\sigma_{e}^{B} \omega_{e}-\sigma_{e}^{B} \omega_{M n}(z)\right)\right)\right]
\end{aligned}
$$

with

$$
\begin{aligned}
\mathbf{s}_{>/<}^{x / \perp} & :=\sum_{\mathbf{k}}^{>/<} \mathbf{s}_{\mathbf{k}}^{x / \perp} \\
\Gamma^{>/<}(z): & =\sum_{\mathbf{k}}^{>/<} \Gamma(\mathbf{k}, z) \\
\mathbf{S}^{x / \perp,(j)} & :=d^{j-1} \int_{-\frac{d}{2}}^{\frac{d}{2}} d z|\psi(z)|^{2 j}\left\langle\mathbf{S}^{x / \perp}(z)\right\rangle
\end{aligned}
$$

where the indices $x$ and $\perp$ denote the spin components parallel and perpendicular to the equilibrium axis $x$ and $\sum_{\mathbf{k}}{ }^{>/<}$describes the sum over all wave vectors $\mathbf{k}$ with $\omega_{\mathbf{k}}>\omega_{0}$ or $\omega_{\mathbf{k}}<\omega_{0}$, respectively, where $\omega_{0}=\mid \sigma_{e}^{B} \omega_{e}-$ $\sigma_{M n}^{B} \omega_{M n} \mid$. The distinction between states with higher or lower kinetic energy than $\omega_{0}$ is a direct consequence of the step-like $\mathbf{k}$ dependence of the spin transfer rates.

Eqs. (49) of the present paper differ mainly from Eqs. (4) and (5) of Ref. 39 in that carriers with $\omega_{\mathbf{k}}<\omega_{0}$ are distinguished from carriers with $\omega_{\mathbf{k}}>\omega_{0}$ and in that the terms proportional to the rate $\Gamma^{>/<}(z)$ are omitted in the mean-field treatment of Ref. 39. Instead, a phenomenological relaxation rate $\tau_{e}^{-1}$ was added manually in Ref. 39. Another difference is the appearance of the corresponding spin transfer term in the equations for the impurities. This is due to the fact that the $s$ - $d$ interaction is spin conserving so that the electron spin that is removed from $\mathbf{s}_{>/<}^{\perp}$ has to be transferred to the impurity system. Taking these corrections with respect to the description of Ref. 39 into account would lead to a more accurate modelling of the collective carrier-impurity pre- 
cession modes. However, as discussed earlier, the variation of the perpendicular spin transfer rate in the presence of an external magnetic field is limited to $\lesssim 30 \%$ of the golden rule value at $\mathbf{B}=0$, so that the spin transfer rate remains in the same order of magnitude. Thus, the phenomenological treatment of the rate can be justified for the purpose of the discussion in Ref. 39.

\section{CONCLUSION}

A quantum kinetic description of the carrier spin dynamics in paramagnetic intrinsic II-VI DMS was presented which, in contrast to previous works ${ }^{33,36,37}$, also accounts for a wave-vector dependent effective magnetic field as well as Zeeman terms for carriers and impurities. The Markov limit of the quantum kinetic equations allow us to extract rates for spin transfer processes between carriers and magnetic impurities. From the rigorous treatment of a precession-type dynamics of the carrier-impurity correlations it is found that the redistribution of carriers in $\mathbf{k}$-space is not only influenced by the spin-splitting of the electron subbands due to the Zeeman energy enhanced by the impurity magnetization, but also acquires an energetic shift corresponding to the Zeeman level splitting of the magnetic impurities. This shift accounts for the fact that a spin flip of an electron involves a spin flop of the magnetic impurity in the opposite direction and the total energy of the magnetic impurity and the electon spin has to be conserved. The energetic shifts in the description of the spin flip-flop processes are often not correctly accounted for in the literature.

The impact of these energy shifts was investigated using the example of the magnetic-field dependence of the carrier-impurity spin transfer rates parallel and perpendicular to the impurity magnetization. Two distinct parameter regimes were identified, one for rather high doping concentrations of the order of $x \sim 1 \%$ and one for extremely diluted systems with $x \lesssim 0.1 \%$. These regimes correspond to cases where the total change of the kinetic electron energy as given by $\left(\sigma_{e}^{B} \omega_{e}-\sigma_{M n}^{B} \omega_{M n}\right)$ is mainly positive or negative. In both situations the perpendicular spin transfer rate $T_{2}^{-1}$ varies within $\sim 30 \%$ of the value for $B=0$, which also coincides with the results for $T_{1}^{-1}$ obtained by Fermi's golden rule. However, in the first case, the parallel spin transfer rate $T_{1}^{-1}$ decays monotonically for an increasing magnetic field to $\frac{5}{8}$ of the Golden Rule value at $B=0$, while in the extremely diluted case, $T_{1}^{-1}$ eventually vanishes. In calculations where the carrier spin splitting $\hbar \omega_{e}$ or the impurity Zeeman splitting $\hbar \omega_{M n}$ is neglected, as is often done in the literature, the magnetic-field dependence of the spin transfer rates deviates significantly from that predicted by the accurate description involving both energetic shifts. Accounting for the impurity Zeeman splitting for the spin flip-flop processes turns out to be particularly important in the very dilute case.
Furthermore, the interplay between the $s$ - $d$ interaction between carrier and impurites and the Rashba interaction in a $\mathrm{Hg}_{1-x-y} \mathrm{Cd}_{y} \mathrm{Mn}_{x}$ Te quantum well was investigated. In the standard rate description approach one usually calculates for each interaction a corresponding scattering rate and ignores that other interactions might change the dynamics during the scattering process. This was the point of view adopted in previous studies of the combined dynamics of $s-d$ and Rashba couplings ${ }^{21,49}$. However, such mutual dependencies of different interactions have been shown in the literature to be of importance, e.g., in the case of a static electric field acting during phonon scattering process known as intracollisional field effects $^{38}$. Technically, the dynamics during an ongoing interaction process is represented by correlation functions. In the present article, we presented a quantum kinetic description where $s$ - $d$ and Rashba interactions have been fully accounted for in the combined dynamics of the single-particle density matrices and the carrier-impurity correlations, thus fully covering all mutual cross-effects between these interactions. While it is a priori difficult to predict how important these cross-effects actually are, we have demonstrated for the present case that the total carrier spin is hardly affected by this mechanism.

Finally, taking into account also the $z$-dependence of the carrier envelope function makes it possible to show how the phenomenological treatment of the spin transfer rate in the description of collective carrier-impurity precession modes in Ref. 39 can be based on a solid microscopic foundation.

In summary, our microscopic treatment of the effects of a k-dependent magnetic field and the impact of the shape of the carrier envelope function justifies the approximations made in earlier studies of the dynamics of the total electron $\operatorname{spin}^{21,39}$. Apart from this new insight, the present theory further contributes to the progress in the field of spin physics in DMS by not only deriving rates for carrier spins parallel, but also perpendicular to the impurity magnetization in the presence of an external magnetic field. The latter are expected to be the dominant contribution to the carrier dephasing time in time-resolved magneto-optical Kerr measurements in Voigt configuration. In contrast to earlier approaches found in the literature ${ }^{22,32}$, the rates derived in this article are fully compatible with the energy conservation of an individual spin flip-flop process. Our study reveals that the difference between the predictions of the discussed theories is most prominent for extremely diluted magnetic semiconductors.

\section{ACKNOWLEDGMENTS}

We gratefully acknowledge the financial support from the Universidad de Buenos Aires, project UBACyT 20142017 No. 20020130100514BA, and from CONICET, project PIP 11220110100091. 
1 T. Dietl and H. Ohno, Rev. Mod. Phys. 86, 187 (2014).

2 T. Dietl, H. Ohno, F. Matsukura, J. Cibert, and D. Ferrand, Science 287, 1019 (2000).

3 Z. Ben Cheikh, S. Cronenberger, M. Vladimirova, D. Scalbert, F. Perez, and T. Wojtowicz, Phys. Rev. B 88, 201306 (2013).

4 Y. S. Chen, M. Wiater, G. Karczewski, T. Wojtowicz, and G. Bacher, Phys. Rev. B 87, 155301 (2013).

5 S. Cronenberger, M. Vladimirova, S. V. Andreev, M. B. Lifshits, and D. Scalbert, Phys. Rev. Lett. 110, 077403 (2013).

${ }^{6}$ F. Perez, J. Cibert, M. Vladimirova, and D. Scalbert, Phys. Rev. B 83, 075311 (2011).

7 M. D. Kapetanakis, I. E. Perakis, K. J. Wickey, C. Piermarocchi, and J. Wang, Phys. Rev. Lett. 103, 047404 (2009).

8 M. D. Kapetanakis and I. E. Perakis, Phys. Rev. Lett. 101, 097201 (2008).

${ }^{9}$ H. Krenn, K. Kaltenegger, T. Dietl, J. Spałek, and G. Bauer, Phys. Rev. B 39, 10918 (1989).

10 S. A. Crooker, D. D. Awschalom, J. J. Baumberg, F. Flack, and N. Samarth, Phys. Rev. B 56, 7574 (1997).

11 P. Barate, S. Cronenberger, M. Vladimirova, D. Scalbert, F. Perez, J. Gòmez, B. Jusserand, H. Boukari, D. Ferrand, H. Mariette, J. Cibert, and M. Nawrocki, Phys. Rev. B 82, 075306 (2010).

12 I. Žutić, J. Fabian, and S. Das Sarma, Rev. Mod. Phys. 76, 323 (2004)

13 T. Dietl, Nat. Mater. 9, 965974 (2010).

14 H. Ohno, Nat. Mater. 9, 952 (2010).

15 A. Haury, A. Wasiela, A. Arnoult, J. Cibert, S. Tatarenko, T. Dietl, and Y. M. d'Aubigné, Phys. Rev. Lett. 79, 511 (1997).

16 M. D. Kapetanakis, J. Wang, and I. E. Perakis, J. Opt. Soc. Am. B 29, A95 (2012).

17 T. Jungwirth, J. Sinova, J. Mašek, J. Kučera, and A. H. MacDonald, Rev. Mod. Phys. 78, 809 (2006).

18 A. Patz, T. Li, X. Liu, J. K. Furdyna, I. E. Perakis, and J. Wang, Phys. Rev. B 91, 155108 (2015).

19 Y. A. Bychkov and E. I. Rashba, Journal of Physics C: Solid State Physics 17, 6039 (1984).

20 G. Dresselhaus, Phys. Rev. 100, 580 (1955).

21 F. Ungar, M. Cygorek, P. I. Tamborenea, and V. M. Axt, Phys. Rev. B 91, 195201 (2015).

22 J. H. Jiang, Y. Zhou, T. Korn, C. Schüller, and M. W. Wu, Phys. Rev. B 79, 155201 (2009).

${ }^{23}$ K. E. Rönnburg, E. Mohler, H. G. Roskos, K. Ortner, C. R. Becker, and L. W. Molenkamp, Phys. Rev. Lett. 96, 117203 (2006).

24 J. K. Furdyna, J. Appl. Phys. 64, R29 (1988).

25 B. König, I. A. Merkulov, D. R. Yakovlev, W. Ossau, S. M. Ryabchenko, M. Kutrowski, T. Wojtowicz, G. Karczewski, and J. Kossut, Phys. Rev. B 61, 16870 (2000).

26 O. Morandi, P.-A. Hervieux, and G. Manfredi, Phys. Rev. B 81, 155309 (2010).
27 O. Morandi and P.-A. Hervieux, Phys. Rev. B 81, 195215 (2010).

28 M. Cygorek, P. I. Tamborenea, and V. M. Axt, Phys. Rev. B 93, 035206 (2016).

29 E. Tsitsishvili and H. Kalt, Phys. Rev. B 73, 195402 (2006).

30 E. Tsitsishvili and H. Kalt, Phys. Rev. B 77, 155305 (2008).

31 J. Kossut, physica status solidi (b) 72, 359 (1975).

32 Y. G. Semenov, Phys. Rev. B 67, 115319 (2003).

33 C. Thurn and V. M. Axt, Phys. Rev. B 85, 165203 (2012).

34 C. Thurn, M. Cygorek, V. M. Axt, and T. Kuhn, Phys. Rev. B 87, 205301 (2013).

35 C. Thurn, M. Cygorek, V. M. Axt, and T. Kuhn, Phys. Rev. B 88, 161302(R) (2013).

36 M. Cygorek and V. M. Axt, Phys. Rev. B 90, 035206 (2014).

37 M. Cygorek and V. M. Axt, Semicond. Sci. Technol. 30, 085011 (2015).

38 J. Hader, T. Meier, S. W. Koch, F. Rossi, and N. Linder, Phys. Rev. B 55, 13799 (1997).

39 M. Vladimirova, S. Cronenberger, P. Barate, D. Scalbert, F. J. Teran, and A. P. Dmitriev, Phys. Rev. B 78, 081305 (2008).

40 F. J. Teran, M. Potemski, D. K. Maude, D. Plantier, A. K. Hassan, A. Sachrajda, Z. Wilamowski, J. Jaroszynski, T. Wojtowicz, and G. Karczewski, Phys. Rev. Lett. 91, 077201 (2003).

41 Here, we use the convention that the factor $\hbar$ which appears in the spin matrices in the SI system is instead included in $\mu_{B}$ and $J_{s d}$, respectively.

42 A. D. Margulis and V. A. Margulis, Fiz. Tverda Tela (Leningrad) 25, 1590 (1983), [Sov. Phys. Solid State 25 918 (1983)].

43 N. R. Ogg, Phys. Soc. London 89, 431 (1966).

44 F. X. Bronold, I. Martin, A. Saxena, and D. L. Smith, Phys. Rev. B 66, 233206 (2002).

45 The source terms $c_{l \mathbf{k}_{1}}^{\alpha \mathbf{k}_{2}}$ are given by $c_{l \mathbf{k}_{1}}^{\alpha \mathbf{k}_{2}}:=$ $\sum_{\sigma_{1} \sigma_{2} n_{1} n_{2}} S_{n_{1} n_{2}}^{l} s_{\sigma_{1} \sigma_{2}}^{\alpha} b_{\sigma_{1} n_{1} \mathbf{k}_{1}}^{\sigma_{2} n_{2} \mathbf{k}_{2} I I I}$ with $b_{\sigma_{1} n_{1} \mathbf{k}_{1}}^{\sigma_{2} n_{2} \mathbf{k}_{2} I I I}$ being defined in Ref. 36.

46 F. Rossi and T. Kuhn, Rev. Mod. Phys. 74, 895 (2002).

47 M. Cygorek and V. M. Axt, Journal of Physics: Conference Series 647, 012042 (2015).

48 G. Bastard and R. Ferreira, Surface Science 267, 335 (1992).

49 F. Ungar, M. Cygorek, P. I. Tamborenea, and V. M. Axt, Journal of Physics: Conference Series 647, 012010 (2015).

${ }^{50}$ F. J. Teran, M. Potemski, D. K. Maude, D. Plantier, A. K. Hassan, A. Sachrajda, Z. Wilamowski, J. Jaroszynski, T. Wojtowicz, and G. Karczewski, Phys. Rev. Lett. 91, 077201 (2003).

51 P. M. Shmakov, A. P. Dmitriev, and V. Y. Kachorovskii, Phys. Rev. B 83, 233204 (2011).

52 D. Frustaglia, J. König, and A. H. MacDonald, Phys. Rev. B 70, 045205 (2004). 